\title{
NEW PRECISION ORBITS OF BRIGHT DOUBLE-LINED SPECTROSCOPIC BINARIES. VII. 47 ANDROMEDAE, 38 CASSIOPEIAE, AND HR 8467
}

\author{
Francis C. Fekel ${ }^{1,4}$, Jocelyn Tomkin ${ }^{2}$, Michael H. Williamson ${ }^{1}$, and Dimitri Pourbaix ${ }^{3}$ \\ ${ }^{1}$ Center of Excellence in Information Systems, Tennessee State University, Nashville, TN 37209, USA; fekel@evans.tsuniv.edu \\ ${ }^{2}$ Astronomy Department and McDonald Observatory, University of Texas, Austin, TX 78712, USA; jt@alexis.as.utexas.edu \\ ${ }^{3}$ FNRS Institut d'Astronomie et d'Astrophysique, Université Libre de Bruxelles, CP 226, B-1050 Bruxelles, Belgium; pourbaix@astro.ulb.ac.be \\ Received 2011 April 13; accepted 2011 May 23; published 2011 July 27
}

\begin{abstract}
Improved orbital elements for three double-lined spectroscopic binaries, 47 And, 38 Cas, and HR 8467, have been determined with extensive new radial velocities. For 38 Cas lines of the secondary have been detected for the first time. Given the orbital periods for these systems of 35.3682, 134.130, and 42.3813 days, respectively, it is not surprising that all three have either moderate or relatively high eccentricities. The orbital dimensions $\left(a_{1} \sin i\right.$ and $\left.a_{2} \sin i\right)$ and minimum masses $\left(m_{1} \sin ^{3} i\right.$ and $\left.m_{2} \sin ^{3} i\right)$ have accuracies of $0.5 \%$ or better. An astrometric orbit for 38 Cas, which was recomputed with Hipparcos astrometry and our new spectroscopic orbital elements, produces a very high orbital inclination of $88^{\circ} \pm 5^{\circ}$. We have found no evidence for eclipses in either 38 Cas or HR 8467 . We estimate that both components of $38 \mathrm{Cas}$ are slightly metal poor with $[\mathrm{Fe} / \mathrm{H}]=-0.3$. The two components of 47 And are Am main-sequence stars, while our spectral types for 38 Cas are F6 dwarf and G5 dwarf for its primary and secondary, respectively. For HR 8467 we determined spectral types of F6 subgiant and F6 dwarf for the components. The primary of HR 8467 is likely just beginning to traverse the Hertzsprung gap and is rotating more slowly than its pseudosynchronous velocity, while the main-sequence secondary is rotating pseudosynchronously. On the other hand, the binary components of 47 And and 38 Cas are rotating significantly faster than their pseudosynchronous velocities.
\end{abstract}

Key words: binaries: spectroscopic - stars: fundamental parameters - stars: individual (47 And, 38 Cas, HR 8467)

\section{INTRODUCTION}

Observations of binary stars that can be resolved both spectroscopically and visually enable the direct determination of stellar masses and precise stellar parallaxes from their threedimensional orbits. With the improvement of ground-based optical and near-infrared interferometry over the past two decades, there is now a greatly enhanced overlap of the spectroscopic and visual binary domains (Quirrenbach 2001). Cunha et al. (2007) provided a list of over 30 interferometric visual orbits for doublelined spectroscopic binaries, while Torres et al. (2010) identified 23 interferometric systems with stellar masses determined to better than $3 \%$. As a result, work on individual systems (e.g., Hummel et al. 2001; Boden et al. 2006; Fekel et al. 2009a) has led to useful comparisons with evolutionary theory.

In SB9, the continually updated Web-based edition of the spectroscopic binary orbit catalog (Pourbaix et al. 2004), many of the older orbits have been computed with radial velocities from photographic plates. Because those plates generally have lower resolutions and much lower signal-to-noise ratios than modern CCD observations, results from them limit the precision of three-dimensional orbits. Thus, in previous papers in this series (Tomkin \& Fekel 2006, 2008; Fekel et al. 2009b, 2010, 2011; Fekel \& Williamson 2010) we have obtained new radial velocities and computed spectroscopic orbits for 16 bright field spectroscopic binaries. Because these bright systems are the most accessible to interferometry, the improved spectroscopic orbits should greatly enhance the possibility of

\footnotetext{
4 Visiting Astronomer, Kitt Peak National Observatory, National Optical Astronomy Observatory, operated by the Association of Universities for Research in Astronomy, Inc. under cooperative agreement with the National Science Foundation.
}

interferometric observations of them. Several of the previously analyzed systems are also eclipsing binaries that need additional photometric observations to improve their light curves.

To obtain the individual mass and parallax of a binary system, spectroscopic orbits of the two components are needed. While both 47 And and HR 8467 are previously known double-lined spectroscopic binaries, 38 Cas has only a single-lined orbit in the literature. However, we have detected lines of the $38 \mathrm{Cas}$ secondary component, turning a former single-lined system into a more useful double-lined one. Some basic information about the three systems is given in Table 1 .

\section{BRIEF HISTORY}

$$
\text { 2.1. } 47 \text { And }=H R 395=H D 8374=H I P 6514
$$

In a list of binaries discovered at the Dominion Astrophysical Observatory (DAO), Plaskett \& Young (1919) noted that 47 And $\left[\alpha=01^{\mathrm{h}} 23^{\mathrm{m}} 40^{\mathrm{s}} 62, \delta=37^{\circ} 42^{\prime} 53^{\prime \prime} .8(2000)\right]$ is a doublelined spectroscopic binary. With the help of additional plates obtained at the Yerkes and McDonald Observatories, Jose (1951) estimated values for several orbital elements, including a period of 39.393 days and an eccentricity of approximately 0.6. Abt (1961) observed 47 And as part of a survey of metallic-line A and early-F stars. Unfortunately, only three of his seven spectra were double-lined, and with his limited velocities he was unable to compute a new orbital solution. As a result of a more extensive observing campaign at the DAO, Fletcher (1967) determined a greatly improved orbit that has a substantially revised period of 35.371 days.

Jaschek-Corvalan \& Jaschek (1957) included 47 And in a table of metallic-line stars that were identified by W. Bidelman. Osawa (1959) classified the combined spectrum of 47 And as A3, F2, and F5 for its Ca II K, hydrogen, and metallic lines, 
Table 1

Basic Properties of the Program Stars

\begin{tabular}{lrrcccrr}
\hline \hline Name & HR & HD & Spectral Type & $\begin{array}{c}V^{\mathrm{a}} \\
(\mathrm{mag})\end{array}$ & $\begin{array}{c}B-V^{\mathrm{a}} \\
(\mathrm{mag})\end{array}$ & $\begin{array}{c}\text { Parallax }^{\mathrm{b}} \\
(\mathrm{mas})\end{array}$ & $\begin{array}{r}\text { Period } \\
\text { (days) }\end{array}$ \\
\hline 47 And & 395 & 8374 & Am & 5.60 & 0.276 & 15.66 & 35.37 \\
38 Cas & 427 & 9021 & F6 V & 5.82 & 0.489 & 34.51 & 134.13 \\
$\ldots$ & 8467 & 210763 & F6 V & 6.39 & 0.498 & 11.23 & 42.38 \\
\hline
\end{tabular}

Notes.

a Perryman et al. (1997).

b van Leeuwen (2007).

respectively, while Abt \& Morrell (1995) found similar spectral classes of A1, F1, and F2 for those three line groups. Abt \& Moyd (1973) and Abt \& Morrell (1995) concluded that both components were relatively slowly rotating.

$$
\text { 2.2. } 38 \text { Cas }=H R 427=H D 9021=H I P 7078
$$

The star 38 Cas $\left[\alpha=01^{\mathrm{h}} 31^{\mathrm{m}} 13.76, \delta=70^{\circ} 15^{\prime} 52^{\prime \prime} .6\right.$ (2000)] was first brought to prominence when Adams et al. (1924) included it in a list of 97 stars having variable velocity. From velocity measurements of eight Mount Wilson plates, which had a range of $31 \mathrm{~km} \mathrm{~s}^{-1}$, they concluded that it is a spectroscopic binary. With 44 velocities obtained at the DAO, Wright \& Pugh (1954) determined a single-lined orbit for 38 Cas that has a period of 134.078 days.

For two decades interest in 38 Cas receded until McAlister (1976) produced a list of spectroscopic binaries that might be resolved with a speckle interferometer and large aperture telescopes. He identified $38 \mathrm{Cas}$ as one of a number of singlelined binaries that had estimated nodal separations greater than 0.02 , the limit of resolution of a $5 \mathrm{~m}$ telescope. McAlister (1978) and Hartkopf \& McAlister (1984) used the $4 \mathrm{~m}$ Mayall telescope at the Kitt Peak National Observatory (KPNO) and obtained five speckle observations of 38 Cas between 1975 and 1982. They did not resolve the system on any of those occasions and so concluded that the binary pair had a separation of $<0^{\prime \prime} .031$ at the times of observation. However, with the help of the spectroscopic orbit, the Hipparcos team (Perryman et al. 1997) used the Hipparcos data to determine an astrometric orbit for 38 Cas. More recently, Jancart et al. (2005) recomputed its astrometric orbit from that data set and the spectroscopic elements of Wright \& Pugh (1954). A comparison of the two astrometric solutions shows that the angular separation found by Jancart et al. (2005) is a factor of 1.28 larger than that found by the Hipparcos team. Both analyses indicate that the orbital inclination is close to $90^{\circ}$. The 38 Cas system has been classified as F6 V by Cowley (1976) and Cowley \& Bidelman (1979) as well as more recently by Abt (2009).

\section{3. $H R 8467=H D 210763=H I P 109647$}

HR $8467\left[\alpha=22^{\mathrm{h}} 12^{\mathrm{m}} 43^{\mathrm{s}} .87, \delta=-04^{\circ} 43^{\prime} 14^{\prime \prime} 4\right.$ (2000)] was one of 2111 stars for which Wilson \& Joy (1950) published radial velocities that were obtained at Mount Wilson Observatory. Their six velocities have a range of $60 \mathrm{~km} \mathrm{~s}^{-1}$, and so they concluded that the star has a variable velocity. Later, Abt (1973) published information concerning the individual spectrograms. Between 1972 and 1981 at Haute Provence Observatory Nadal et al. (1983) obtained an additional 45 observations, 31 of which showed two sets of lines. From their velocities they published a double-lined orbit for which they obtained a period of 42.3813 days and an eccentricity of 0.616. Nadal et al. (1983) also suggested that the inclination of the system was close to $90^{\circ}$, and so, eclipses are a possibility. Harlan (1974) classified HR 8467 as F8 IV, while Malaroda (1975) considered it to be F7 V and Cowley (1976) called it F6 V.

\section{OBSERVATIONS AND RADIAL VELOCITIES}

We obtained spectroscopic observations of the binaries at three observatories. From 2003 through 2011, we collected an extensive number of spectra of the three systems with the Tennessee State University (TSU) $2 \mathrm{~m}$ automatic spectroscopic telescope (AST), a fiber-fed echelle spectrograph, and a $2048 \times$ 4096 SITe ST-002A CCD. The echelle spectrograms have 21 orders that cover the wavelength range 4920-7100 $\AA$ with an average resolution of $0.17 \AA$. The typical signal-to-noise ratio of these observations is $\sim 80$. Eaton \& Williamson (2004, 2007) have given a more extensive description of the telescope and spectrograph, operated at Fairborn Observatory near Washington Camp in the Patagonia Mountains of southeastern Arizona.

From 2003 through 2010, we also obtained spectrograms of the program stars at KPNO with the coudé feed telescope and coudé spectrograph. Nearly all observations were made with a Texas Instruments (TI) CCD detector, and those spectra are centered at $6430 \AA$, cover a wavelength range of $84 \AA$, and have a resolution of $0.21 \AA$ or a resolving power of just over 30,000 . However, in 2008 September the TI detector was unavailable, and so we used a Tektronics CCD, designated T1KA. With that CCD the spectrum was centered at $6400 \AA$. The wavelength coverage was increased to $172 \AA$, but the resolving power decreased to 19,000 . In 2010 September the TI CCD was retired, and instead a new CCD, made by Semiconductor Technology Associates and designated STA2, was used. It consists of a $2600 \times 4000$ array of $12 \mu \mathrm{m}$ pixels. With STA2 the spectrum once again was centered at $6430 \AA$, and the size of the detector produced a wavelength range of $336 \AA$. The spectrograph slit was set so that the STA2 spectra have the same resolution as those acquired with the TI CCD.

Finally, from 2004 through 2006 we obtained spectra of the three systems at McDonald Observatory with the $2.1 \mathrm{~m}$ telescope, the Sandiford Cassegrain echelle spectrograph (McCarthy et al. 1993), and a Reticon CCD. The spectrograms cover the wavelength range 5700-7000 $\AA$ and have a resolving power of 49,000. Further details about our KPNO and McDonald observations are given in Tomkin \& Fekel (2006).

Fekel et al. (2009b) provided an extensive general description of velocity measurement of the Fairborn AST spectra. Here we mention that for 47 And, 38 Cas, and HR 8467 we used a line list for solar-type stars and that the resulting Fairborn velocities are absolute velocities. Our unpublished measurements of several IAU standard solar-type stars indicate that the Fairborn Observatory velocities have a small zero-point offset of $-0.3 \mathrm{~km} \mathrm{~s}^{-1}$ relative to the velocities of Scarfe et al. (1990). Thus, we have added $0.3 \mathrm{~km} \mathrm{~s}^{-1}$ to each Fairborn velocity.

The procedures used to measure the McDonald and KPNO radial velocities have been described extensively in Tomkin \& Fekel (2006). We note here that the McDonald velocities are absolute velocities, which were placed on a secure rest scale by means of the telluric $\mathrm{O}_{2}$ and $\mathrm{H}_{2} \mathrm{O}$ lines in the stellar spectra. On the other hand, the KPNO velocities are relative velocities, being determined by cross-correlation with respect to IAU radial velocity standard stars of the same or similar spectral type as 


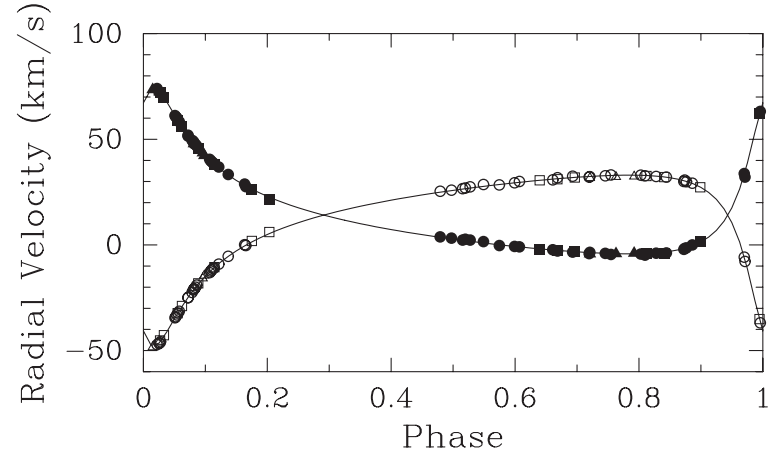

Figure 1. Radial velocities of 47 And compared with the computed velocity curves. Filled and open symbols represent the primary and secondary, respectively. Circles: Fairborn Observatory; triangles: KPNO; squares: McDonald Observatory. Zero phase is a time of periastron passage.

the program stars. The velocities adopted for those standards are from Scarfe et al. (1990).

We used the same procedure outlined by Tomkin \& Fekel (2006) to confirm that no additional offset should be added to the KPNO relative velocities. Among our six components of the three systems, we found no systematic velocity offset for the KPNO velocities and confirm that the KPNO velocities are on the same scale as the absolute velocities of Fairborn and McDonald to within a precision of $0.1-0.2 \mathrm{~km} \mathrm{~s}^{-1}$, as was found by Tomkin \& Fekel (2006). Even if there were such a modest offset, it would simply slightly increase the uncertainty of the orbital elements since the number of Fairborn velocities of each system is at least three times larger.

\section{DETERMINATION OF SPECTROSCOPIC ORBITS AND RESULTS}

We have used several computer programs to determine the orbital elements. Preliminary orbits were computed with the program BISP (Wolfe et al. 1967), which implements a slightly modified version of the Wilsing-Russell method. Eccentric orbits were then determined with SB1 (Barker et al. 1967), a program that uses differential corrections. For a simultaneous solution of the two components, we used SB2, which is a slightly modified version of SB1.

The spectra that we obtained at the different observatories have different wavelength ranges. Thus, different numbers of lines were available for measurement. In addition, the primary and secondary lines are of different quality because the components differ in strength and in line width. Thus, the velocity precision of each set of data will vary from observatory to observatory, and the precision will also usually differ for each primary and secondary component. To determine the weight for each set of our velocities, as well as those from the literature, we computed the variances of the individual orbital solutions, which are inversely proportional to our adopted weights.

\subsection{And}

At Fairborn Observatory between 2004 and 2011 we acquired 53 spectrograms, all of which show double lines. An additional 15 observations from McDonald Observatory and 8 more from KPNO were also obtained. All our velocities are listed in Table 2. Because of the modest number of McDonald velocities and the even smaller number from KPNO, coverage at important phases of the eccentric orbit is limited in those two data sets, and so velocities from the two observatories

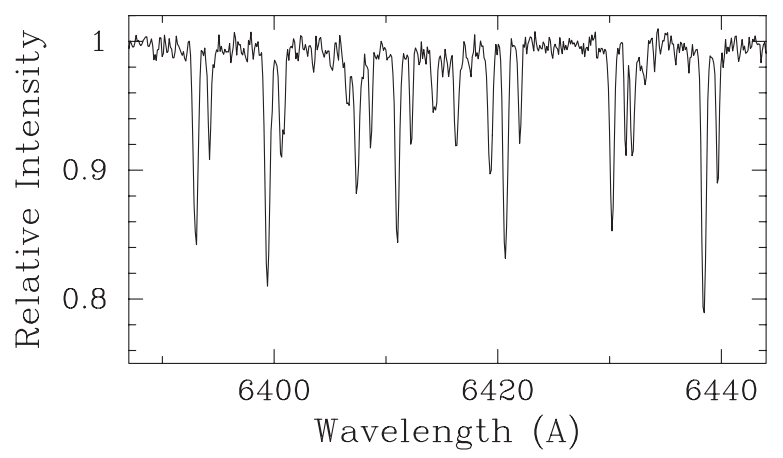

Figure 2. Spectrum of $38 \mathrm{Cas}$ in the $6430 \AA$ region. The weaker, redshifted, secondary components are very obvious.

have been combined in the initial orbital solutions that we have obtained. Because the Fairborn velocities are numerous and well distributed in phase, those velocities were analyzed separately. Thus, we first computed four single-lined orbital solutions, two for each component. Examining those four orbits, the secondary velocities from Fairborn Observatory were the most precise, and so those velocities were given weights of 1.0. Relative weights for the other velocity sets were 0.7 for the McDonald and KPNO primary, and 0.6 for the McDonald and KPNO secondary as well as for the Fairborn primary. The four individual solutions have center-of-mass velocities within $0.3 \mathrm{~km} \mathrm{~s}^{-1}$ of each other. Thus, we computed a simultaneous solution of the weighted primary and secondary velocities from the three observatories, which produced a period of 35.3682 days.

In principle, the earlier radial velocities of 47 And could be used to improve the precision of the period and possibly the other elements. Thus, we examined the DAO velocities of Fletcher (1967), obtaining individual orbital solutions for his primary and secondary velocities. We found the primary velocities to have significantly lower residuals, and so, only those velocities with weights of 0.06 were combined with our new velocities into a double-lined orbital solution. In this new solution only the uncertainty of the period was improved, but because most of the DAO velocities have large negative residuals, and the period change was less than $2 \sigma$, we have chosen not to include the DAO velocities in our final results, so we adopt the simultaneous solution of our new primary and secondary velocities, which is listed in Table 3. Figure 1 compares our primary and secondary velocities with the calculated velocity curves. Zero phase is a time of periastron passage.

In addition to our new elements, Table 3 lists the elements of Fletcher (1967). A comparison shows that while values of the elements are generally in good agreement, our new elements are 5-10 times more precise. The systemic velocities of the two solutions differ by less than $1 \mathrm{~km} \mathrm{~s}^{-1}$, so there is no velocity evidence for a third component in the system.

\subsection{Cas}

Between 2003 and 2011, we obtained spectroscopic observations of 38 Cas at three observatories. At Fairborn Observatory we acquired 54 double-lined spectrograms, while the number at KPNO was just 16 and at McDonald Observatory, only 4 (Table 4). A portion of a KPNO red-wavelength spectrum plotted in Figure 2 shows lines from the two components. Using the Fairborn velocities, we determined individual orbital solutions for the primary and secondary. Because of the very small number McDonald velocities, we added them to the KPNO velocity set and determined orbital solutions for the two components 
Table 2

Radial Velocities of 47 And

\begin{tabular}{|c|c|c|c|c|c|c|c|c|}
\hline $\begin{array}{l}\text { Hel. Julian Date } \\
\text { (HJD - 2,400,000) }\end{array}$ & Phase & $\begin{array}{c}V_{1} \\
\left(\mathrm{~km} \mathrm{~s}^{-1}\right)\end{array}$ & $\begin{array}{l}(O-C)_{1} \\
\left(\mathrm{~km} \mathrm{~s}^{-1}\right)\end{array}$ & $\mathrm{Wt}_{1}$ & $\begin{array}{c}V_{2} \\
\left(\mathrm{~km} \mathrm{~s}^{-1}\right)\end{array}$ & $\begin{array}{c}(O-C)_{2} \\
\left(\mathrm{~km} \mathrm{~s}^{-1}\right)\end{array}$ & $\mathrm{Wt}_{2}$ & Source $^{\mathrm{a}}$ \\
\hline $52,966.818$ & 0.497 & 3.2 & 0.3 & 0.6 & 25.9 & 0.2 & 1.0 & Fair \\
\hline $52,967.886$ & 0.528 & 2.3 & 0.6 & 0.6 & 27.5 & 0.6 & 1.0 & Fair \\
\hline $53,286.959$ & 0.549 & 1.6 & 0.7 & 0.6 & 30.6 & 0.0 & 0.6 & Fair \\
\hline $53,292.997$ & 0.720 & -4.1 & -0.5 & 0.6 & 31.1 & -0.2 & 0.6 & Fair \\
\hline $53,042.595$ & 0.640 & -2.0 & -0.1 & 0.7 & 31.9 & -0.1 & 0.6 & $\mathrm{McD}$ \\
\hline $53,043.602$ & 0.668 & -2.7 & -0.1 & 0.7 & 32.6 & -0.3 & 0.6 & McD \\
\hline $53,044.595$ & 0.696 & -3.3 & -0.1 & 0.7 & 32.1 & -0.1 & 0.6 & $\mathrm{McD}$ \\
\hline $53,260.900$ & 0.812 & -4.1 & 0.0 & 0.7 & 28.6 & 0.9 & 1.0 & $\mathrm{McD}$ \\
\hline $53,261.909$ & 0.841 & -3.9 & -0.5 & 0.7 & 32.1 & -0.3 & 1.0 & $\mathrm{McD}$ \\
\hline $53,301.893$ & 0.971 & 32.1 & -2.6 & 0.6 & -7.8 & -0.7 & 1.0 & Fair \\
\hline $53,319.856$ & 0.479 & 3.8 & 0.2 & 0.6 & 25.4 & 0.4 & 1.0 & Fair \\
\hline $53,333.814$ & 0.874 & -1.7 & -0.1 & 0.6 & 30.7 & 0.4 & 1.0 & Fair \\
\hline $53,334.708$ & 0.899 & 1.4 & 0.1 & 0.7 & 27.3 & -0.1 & 0.6 & $\mathrm{McD}$ \\
\hline $53,340.832$ & 0.072 & 51.9 & 0.1 & 0.6 & -24.9 & -0.2 & 1.0 & Fair \\
\hline $53,359.752$ & 0.607 & -0.9 & 0.0 & 0.6 & 30.0 & 0.4 & 1.0 & Fair \\
\hline $53,662.783$ & 0.175 & 26.2 & -0.2 & 0.7 & 1.9 & 0.5 & 0.6 & $\mathrm{McD}$ \\
\hline $53,663.781$ & 0.203 & 21.7 & -0.9 & 0.7 & 6.1 & 0.8 & 0.6 & $\mathrm{McD}$ \\
\hline $53,676.916$ & 0.575 & -0.3 & -0.4 & 0.6 & 28.4 & -0.2 & 1.0 & Fair \\
\hline $53,691.768$ & 0.995 & 62.3 & 0.4 & 0.7 & 26.9 & 0.3 & 1.0 & $\mathrm{McD}$ \\
\hline $53,745.706$ & 0.520 & 2.7 & 0.7 & 0.6 & -35.1 & 0.1 & 0.6 & Fair \\
\hline $54,001.890$ & 0.763 & -4.1 & 0.0 & 0.7 & 32.2 & -0.7 & 0.6 & KPNO \\
\hline $54,002.928$ & 0.792 & -3.9 & 0.3 & 0.7 & 32.4 & -0.6 & 0.6 & KPNO \\
\hline $54,046.592$ & 0.027 & 72.3 & 0.0 & 0.7 & -45.2 & 0.7 & 0.6 & $\mathrm{McD}$ \\
\hline $54,046.816$ & 0.033 & 69.7 & -0.1 & 0.7 & -42.7 & 0.6 & 0.6 & $\mathrm{McD}$ \\
\hline $54,047.599$ & 0.055 & 59.1 & -0.1 & 0.7 & -32.3 & 0.1 & 0.6 & $\mathrm{McD}$ \\
\hline $54,047.846$ & 0.062 & 55.9 & -0.1 & 0.7 & -28.9 & 0.2 & 0.6 & $\mathrm{McD}$ \\
\hline $54,048.773$ & 0.089 & 45.6 & -0.1 & 0.7 & -18.2 & 0.3 & 0.6 & $\mathrm{McD}$ \\
\hline $54,049.691$ & 0.114 & 38.2 & 0.0 & 0.7 & -10.5 & 0.2 & 0.6 & $\mathrm{McD}$ \\
\hline $54,364.868$ & 0.026 & 72.5 & -0.1 & 0.7 & -45.8 & 0.5 & 0.6 & KPNO \\
\hline $54,366.836$ & 0.081 & 47.9 & -0.3 & 0.7 & -20.4 & 0.7 & 0.6 & KPNO \\
\hline $54,861.660$ & 0.072 & 51.5 & -0.3 & 0.6 & -25.0 & -0.2 & 1.0 & Fair \\
\hline $55,003.973$ & 0.096 & 43.6 & 0.2 & 0.7 & -15.8 & 0.3 & 0.6 & KPNO \\
\hline $55,094.966$ & 0.669 & -2.9 & -0.3 & 0.6 & 31.8 & 0.5 & 1.0 & Fair \\
\hline $55,095.826$ & 0.693 & -3.4 & -0.3 & 0.6 & 32.5 & 0.6 & 1.0 & Fair \\
\hline $55,096.813$ & 0.721 & -4.2 & -0.6 & 0.7 & 31.7 & -0.7 & 0.6 & KPNO \\
\hline $55,099.960$ & 0.810 & -4.8 & -0.7 & 0.6 & 32.7 & -0.2 & 1.0 & Fair \\
\hline $55,124.915$ & 0.515 & 2.3 & 0.1 & 0.6 & 26.6 & 0.2 & 1.0 & Fair \\
\hline $55,144.940$ & 0.082 & 47.9 & -0.3 & 0.6 & -21.0 & 0.0 & 1.0 & Fair \\
\hline $55,242.649$ & 0.844 & -3.8 & -0.5 & 0.6 & 32.1 & 0.0 & 1.0 & Fair \\
\hline $55,243.649$ & 0.872 & -2.1 & -0.4 & 0.6 & 30.0 & -0.4 & 1.0 & Fair \\
\hline $55,456.004$ & 0.877 & -1.4 & -0.1 & 0.6 & 29.9 & -0.2 & 1.0 & Fair \\
\hline $55,460.899$ & 0.015 & 73.5 & -0.7 & 0.7 & -48.4 & -0.5 & 0.6 & KPNO \\
\hline $55,463.841$ & 0.098 & 42.3 & -0.4 & 0.7 & -14.5 & 0.9 & 0.6 & KPNO \\
\hline $55,483.744$ & 0.661 & -2.3 & 0.1 & 0.6 & 30.8 & -0.3 & 1.0 & Fair \\
\hline $55,485.854$ & 0.721 & -3.5 & 0.1 & 0.6 & 32.3 & -0.1 & 1.0 & Fair \\
\hline $55,486.738$ & 0.746 & -4.0 & 0.0 & 0.6 & 32.7 & -0.1 & 1.0 & Fair \\
\hline $55,488.767$ & 0.803 & -4.3 & -0.1 & 0.6 & 33.0 & 0.0 & 1.0 & Fair \\
\hline $55,489.647$ & 0.828 & -3.9 & -0.1 & 0.6 & 32.4 & -0.2 & 1.0 & Fair \\
\hline $55,491.711$ & 0.886 & 0.0 & 0.4 & 0.6 & 29.2 & 0.1 & 1.0 & Fair \\
\hline $55,494.679$ & 0.970 & 33.8 & 0.3 & 0.6 & -5.8 & 0.1 & 1.0 & Fair \\
\hline $55,495.588$ & 0.996 & 63.2 & 0.0 & 0.6 & -36.9 & -0.3 & 1.0 & Fair \\
\hline $55,496.642$ & 0.026 & 72.3 & -0.4 & 0.6 & -46.5 & -0.2 & 1.0 & Fair \\
\hline $55,496.656$ & 0.026 & 72.2 & -0.3 & 0.6 & -46.5 & -0.3 & 1.0 & Fair \\
\hline $55,496.712$ & 0.028 & 71.9 & -0.1 & 0.6 & -45.8 & -0.2 & 1.0 & Fair \\
\hline $55,497.585$ & 0.052 & 60.6 & 0.0 & 0.6 & -34.0 & -0.1 & 1.0 & Fair \\
\hline $55,497.642$ & 0.054 & 60.2 & 0.3 & 0.6 & -33.1 & 0.0 & 1.0 & Fair \\
\hline $55,497.712$ & 0.056 & 58.9 & 0.0 & 0.6 & -32.2 & -0.1 & 1.0 & Fair \\
\hline $55,497.782$ & 0.058 & 58.3 & 0.3 & 0.6 & -31.4 & -0.2 & 1.0 & Fair \\
\hline $55,498.585$ & 0.081 & 49.0 & 0.5 & 0.6 & -21.3 & 0.1 & 1.0 & Fair \\
\hline $55,498.642$ & 0.082 & 48.2 & 0.2 & 0.6 & -20.8 & 0.0 & 1.0 & Fair \\
\hline $55,498.712$ & 0.084 & 47.6 & 0.3 & 0.6 & -20.0 & 0.1 & 1.0 & Fair \\
\hline $55,498.782$ & 0.086 & 46.8 & 0.2 & 0.6 & -19.0 & 0.4 & 1.0 & Fair \\
\hline $55,499.584$ & 0.109 & 39.7 & 0.0 & 0.6 & -12.4 & -0.2 & 1.0 & Fair \\
\hline $55,499.642$ & 0.110 & 39.4 & 0.2 & 0.6 & -11.8 & 0.0 & 1.0 & Fair \\
\hline $55,499.712$ & 0.112 & 38.8 & 0.1 & 0.6 & -11.2 & 0.1 & 1.0 & Fair \\
\hline
\end{tabular}


Table 2

(Continued)

\begin{tabular}{lccccrrrr}
\hline \hline $\begin{array}{l}\text { Hel. Julian Date } \\
(\mathrm{HJD}-2,400,000)\end{array}$ & Phase & $\begin{array}{c}V_{1} \\
\left(\mathrm{~km} \mathrm{~s}^{-1}\right)\end{array}$ & $\begin{array}{c}(O-C)_{1} \\
\left(\mathrm{~km} \mathrm{~s}^{-1}\right)\end{array}$ & $\mathrm{Wt}_{1}$ & $\begin{array}{c}V_{2} \\
\left(\mathrm{~km} \mathrm{~s}^{-1}\right)\end{array}$ & $\begin{array}{r}(O-C)_{2} \\
\left(\mathrm{~km} \mathrm{~s}^{-1}\right)\end{array}$ & $\mathrm{Wt}_{2}$ & Source $^{\mathrm{a}}$ \\
\hline $55,499.782$ & 0.114 & 38.4 & 0.2 & 0.6 & -10.8 & -0.1 & 1.0 & Fair \\
$55,500.583$ & 0.137 & 33.3 & 0.3 & 0.6 & -5.4 & 0.0 & 1.0 & Fair \\
$55,501.583$ & 0.165 & 27.6 & -0.3 & 0.6 & -0.3 & -0.2 & 1.0 & Fair \\
$55,557.804$ & 0.755 & -4.5 & -0.4 & 0.6 & 33.1 & 0.2 & 1.0 & Fair \\
$55,570.773$ & 0.122 & 36.9 & 0.5 & 0.6 & -9.1 & -0.2 & 1.0 & Fair \\
$55,587.666$ & 0.599 & -0.7 & 0.0 & 0.6 & 29.4 & 0.0 & 1.0 & Fair \\
$55,602.620$ & 0.022 & 74.0 & 0.4 & 0.6 & -47.2 & 0.1 & 1.0 & Fair \\
$55,603.645$ & 0.051 & 61.2 & 0.0 & 0.6 & -34.5 & 0.0 & 1.0 & Fair \\
$55,604.621$ & 0.079 & 49.5 & 0.2 & 0.6 & -22.5 & -0.3 & 1.0 & Fair \\
$55,605.620$ & 0.107 & 40.5 & 0.3 & 0.6 & -13.1 & -0.3 & 1.0 & Fair \\
$55,607.620$ & 0.163 & 28.8 & 0.6 & 0.6 & 0.1 & 0.6 & 1.0 & Fair \\
\hline
\end{tabular}

Note. ${ }^{a}$ Fair: Fairborn Observatory; KPNO: Kitt Peak National Observatory; McD: McDonald Observatory.

Table 3

Orbital Elements and Related Parameters of 47 And

\begin{tabular}{lcl}
\hline \hline Parameter & Fletcher $(1967)$ & This Study \\
\hline$P($ days $)$ & 35.371 & $35.36815 \pm 0.00015$ \\
$T$ (HJD) & $2,421,894.691$ & $2,454,293.2187 \pm 0.0056$ \\
$e$ & $0.627 \pm 0.010$ & $0.64781 \pm 0.00090$ \\
$\omega_{1}(\mathrm{deg})$ & $320.1 \pm 1.8$ & $325.30 \pm 0.15$ \\
$K_{1}\left(\mathrm{~km} \mathrm{~s}^{-1}\right)$ & $39.0 \pm 0.4$ & $39.216 \pm 0.079$ \\
$K_{2}\left(\mathrm{~km} \mathrm{~s}^{-1}\right)$ & $40.5 \pm 0.4$ & $40.47 \pm 0.073$ \\
$\gamma\left(\mathrm{km} \mathrm{s}^{-1}\right)$ & $13.3 \pm 0.3$ & $14.110 \pm 0.035$ \\
$m_{1} \sin ^{3} i\left(M_{\odot}\right)$ & $0.444 \pm 0.033$ & $0.4172 \pm 0.0020$ \\
$m_{2} \sin ^{3} i\left(M_{\odot}\right)$ & $0.429 \pm 0.033$ & $0.4043 \pm 0.0020$ \\
$a_{1} \sin i\left(10^{6} \mathrm{~km}\right)$ & $14.8 \pm 0.3$ & $14.530 \pm 0.032$ \\
$a_{2} \sin i\left(10^{6} \mathrm{~km}\right)$ & $15.3 \pm 0.3$ & $14.993 \pm 0.031$ \\
$\operatorname{Standard~error~of~a~unit~weight~observation~}\left(\mathrm{km} \mathrm{s}^{-1}\right)$ & $\cdots$ & 0.34 \\
\hline
\end{tabular}

with the combined data. The variances of the four solutions resulted in weights of $1.0,0.9$, and 0.9 for the primary velocities from Fairborn, KPNO, and McDonald, respectively. For the secondary, the weights were $0.9,0.6$, and 0.6 for the same three respective observatories. The center-of-mass velocities of the four data sets agree to within $0.3 \mathrm{~km} \mathrm{~s}^{-1}$. We next obtained a simultaneous solution of all our velocities, which produced an orbital period of 134.130 days.

By far the best previous velocity data set is that of Wright \& Pugh (1954), but given the orientation of the orbit and the linear dispersion of the spectrograms, $30 \AA \mathrm{mm}^{-1}$ at $\mathrm{H} \gamma$, lines of the two components were blended through much of the orbit around apastron, and the secondary was not detected. Thus, we have not included their velocities in our solution.

The new orbital elements from our double-lined solution are given in Table 5. Our primary and secondary velocities are compared with the computed velocity curves in Figure 3. In that plot, zero phase is a time of periastron passage.

For comparison, we also list in Table 5 the orbital elements of Wright \& Pugh (1954). Although the two orbital periods are in good agreement, the measurement of the unresolved lines in the moderate dispersion spectrograms of Wright \& Pugh (1954) results in a $20 \%$ reduction in the semiamplitude of the primary, and a substantially greater eccentricity. The two center-of-mass velocities differ by $1.9 \mathrm{~km} \mathrm{~s}^{-1}$, but this is primarily caused by the measurement of the unresolved components at apastron, which produces a more negative systemic velocity, rather than evidence for a third component in the system.

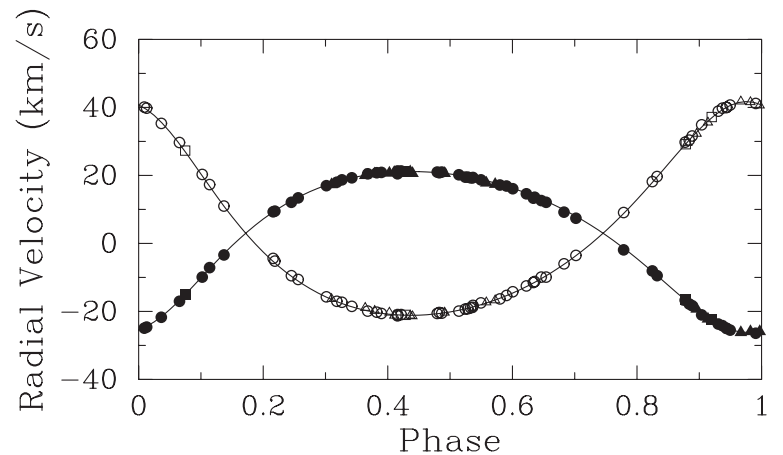

Figure 3. Radial velocities of 38 Cas compared with the computed velocity curves. Filled and open symbols represent the primary and secondary, respectively. Circles: Fairborn Observatory; triangles: KPNO; squares: McDonald Observatory. Zero phase is a time of periastron passage.

\section{3. $H R 8467$}

For HR 8467 we acquired 57 observations at Fairborn Observatory, 16 at KPNO, and 6 at McDonald Observatory between 2003 and 2010 (Table 6). We first obtained orbital solutions for the primary and secondary separately, two for the Fairborn Observatory velocities and because of the small number of McDonald velocities, two for the combined data from KPNO and McDonald Observatory. Comparing the variances of those four solutions, the Fairborn primary velocities are the most precise. Thus, we have adopted weights of 1.0 , 
Table 4

Radial Velocities of $38 \mathrm{Cas}$

\begin{tabular}{|c|c|c|c|c|c|c|c|c|}
\hline $\begin{array}{l}\text { Hel. Julian Date } \\
\text { (HJD - 2,400,000) }\end{array}$ & Phase & $\begin{array}{c}V_{1} \\
\left(\mathrm{~km} \mathrm{~s}^{-1}\right)\end{array}$ & $\begin{array}{l}(O-C)_{1} \\
\left(\mathrm{~km} \mathrm{~s}^{-1}\right)\end{array}$ & $\mathrm{Wt}_{1}$ & $\begin{array}{c}V_{2} \\
\left(\mathrm{~km} \mathrm{~s}^{-1}\right)\end{array}$ & $\begin{array}{c}(O-C)_{2} \\
\left(\mathrm{~km} \mathrm{~s}^{-1}\right)\end{array}$ & $\mathrm{Wt}_{2}$ & Source $^{\mathrm{a}}$ \\
\hline $52,940.756$ & 0.491 & 20.6 & 0.0 & 0.9 & -20.3 & 0.1 & 0.6 & KPNO \\
\hline $53,260.927$ & 0.878 & -16.5 & 0.3 & 0.9 & 29.2 & -0.1 & 0.6 & $\mathrm{McD}$ \\
\hline $53,272.800$ & 0.967 & -26.2 & -0.2 & 0.9 & 41.8 & 0.2 & 0.6 & KPNO \\
\hline $53,274.863$ & 0.982 & -25.9 & 0.2 & 0.9 & 41.6 & -0.1 & 0.6 & KPNO \\
\hline $53,276.858$ & 0.997 & -25.9 & -0.3 & 0.9 & 40.6 & -0.4 & 0.6 & KPNO \\
\hline $53,290.951$ & 0.102 & -9.9 & -0.2 & 1.0 & 20.3 & 0.4 & 0.9 & Fair \\
\hline $53,319.936$ & 0.318 & 18.0 & -0.1 & 1.0 & -17.0 & 0.1 & 0.9 & Fair \\
\hline $53,334.732$ & 0.429 & 21.2 & 0.1 & 0.9 & -21.0 & 0.1 & 0.6 & $\mathrm{McD}$ \\
\hline $53,341.983$ & 0.483 & 20.8 & 0.0 & 1.0 & -20.3 & 0.3 & 0.9 & Fair \\
\hline $53,355.035$ & 0.580 & 17.2 & -0.2 & 1.0 & -16.3 & -0.2 & 0.9 & Fair \\
\hline $53,360.750$ & 0.623 & 14.6 & -0.2 & 1.0 & -12.5 & 0.2 & 0.9 & Fair \\
\hline $53,387.856$ & 0.825 & -8.1 & 0.1 & 1.0 & 18.2 & 0.3 & 0.9 & Fair \\
\hline $53,388.892$ & 0.832 & -9.5 & 0.0 & 1.0 & 19.7 & 0.1 & 0.9 & Fair \\
\hline $53,400.654$ & 0.920 & -22.4 & 0.2 & 0.9 & 37.2 & 0.2 & 0.6 & $\mathrm{McD}$ \\
\hline $53,403.873$ & 0.944 & -25.0 & -0.1 & 1.0 & 40.0 & -0.1 & 0.9 & Fair \\
\hline $53,451.804$ & 0.301 & 17.0 & -0.1 & 1.0 & -15.7 & 0.1 & 0.9 & Fair \\
\hline $53,462.642$ & 0.382 & 20.8 & 0.2 & 1.0 & -20.2 & 0.1 & 0.9 & Fair \\
\hline $53,481.710$ & 0.524 & 19.5 & -0.2 & 1.0 & -19.3 & -0.1 & 0.9 & Fair \\
\hline $53,496.669$ & 0.636 & 13.6 & -0.2 & 1.0 & -11.2 & 0.2 & 0.9 & Fair \\
\hline $53,533.952$ & 0.914 & -22.2 & -0.4 & 0.9 & 35.6 & -0.4 & 0.6 & KPNO \\
\hline $53,649.932$ & 0.778 & -1.9 & -0.5 & 1.0 & 9.1 & 0.3 & 0.9 & Fair \\
\hline $53,672.880$ & 0.950 & -25.5 & -0.2 & 1.0 & 40.7 & 0.1 & 0.9 & Fair \\
\hline $53,689.670$ & 0.075 & -15.0 & 0.0 & 0.9 & 27.3 & 0.3 & 0.6 & $\mathrm{McD}$ \\
\hline $53,694.927$ & 0.114 & -7.1 & 0.3 & 1.0 & 17.3 & 0.5 & 0.9 & Fair \\
\hline $53,728.961$ & 0.368 & 20.5 & 0.3 & 1.0 & -19.9 & -0.1 & 0.9 & Fair \\
\hline $53,743.935$ & 0.479 & 21.0 & 0.2 & 1.0 & -20.6 & 0.1 & 0.9 & Fair \\
\hline $53,758.853$ & 0.591 & 16.9 & 0.1 & 1.0 & -15.3 & 0.1 & 0.9 & Fair \\
\hline $53,773.803$ & 0.702 & 7.4 & -0.6 & 1.0 & -3.5 & 0.1 & 0.9 & Fair \\
\hline $53,798.814$ & 0.888 & -18.3 & 0.0 & 1.0 & 31.6 & 0.3 & 0.9 & Fair \\
\hline $53,842.739$ & 0.216 & 9.3 & 0.1 & 1.0 & -4.4 & 0.9 & 0.9 & Fair \\
\hline $53,859.689$ & 0.342 & 19.3 & 0.0 & 1.0 & -18.5 & 0.1 & 0.9 & Fair \\
\hline $54,001.900$ & 0.403 & 20.7 & -0.2 & 0.9 & -20.3 & 0.5 & 0.6 & KPNO \\
\hline $54,002.933$ & 0.410 & 20.9 & -0.1 & 0.9 & -21.2 & -0.2 & 0.6 & KPNO \\
\hline $54,006.876$ & 0.440 & 20.6 & -0.6 & 0.9 & -21.4 & -0.3 & 0.6 & KPNO \\
\hline $54,264.956$ & 0.364 & 20.0 & -0.1 & 0.9 & -18.9 & 0.8 & 0.6 & KPNO \\
\hline $54,266.980$ & 0.379 & 20.5 & 0.0 & 0.9 & -19.8 & 0.4 & 0.6 & KPNO \\
\hline $54,408.781$ & 0.436 & 21.1 & -0.1 & 0.9 & -21.2 & -0.1 & 0.6 & KPNO \\
\hline $54,873.920$ & 0.904 & -21.0 & -0.5 & 1.0 & 34.9 & 0.6 & 0.9 & Fair \\
\hline $54,957.701$ & 0.528 & 19.5 & -0.1 & 1.0 & -19.2 & -0.1 & 0.9 & Fair \\
\hline $54,958.693$ & 0.536 & 19.3 & 0.0 & 1.0 & -18.8 & -0.1 & 0.9 & Fair \\
\hline $55,004.989$ & 0.881 & -17.2 & 0.0 & 0.9 & 29.6 & -0.2 & 0.6 & KPNO \\
\hline $55,006.988$ & 0.896 & -19.3 & 0.1 & 0.9 & 32.3 & -0.5 & 0.6 & KPNO \\
\hline $55,053.834$ & 0.245 & 12.1 & -0.4 & 1.0 & -9.5 & 0.2 & 0.9 & Fair \\
\hline $55,076.680$ & 0.416 & 20.5 & -0.6 & 1.0 & -21.3 & -0.3 & 0.9 & Fair \\
\hline $55,089.897$ & 0.514 & 20.2 & 0.2 & 1.0 & -19.9 & -0.2 & 0.9 & Fair \\
\hline $55,092.938$ & 0.537 & 19.4 & 0.1 & 1.0 & -18.2 & 0.5 & 0.9 & Fair \\
\hline $55,095.806$ & 0.558 & 17.8 & -0.6 & 0.9 & -17.3 & 0.2 & 0.6 & KPNO \\
\hline $55,105.937$ & 0.634 & 13.3 & -0.7 & 1.0 & -11.5 & 0.1 & 0.9 & Fair \\
\hline $55,145.760$ & 0.931 & -23.7 & 0.0 & 1.0 & 38.9 & 0.4 & 0.9 & Fair \\
\hline $55,153.807$ & 0.991 & -26.4 & -0.5 & 1.0 & 41.2 & -0.2 & 0.9 & Fair \\
\hline $55,156.807$ & 0.013 & -24.6 & -0.2 & 1.0 & 39.8 & 0.4 & 0.9 & Fair \\
\hline $55,159.960$ & 0.036 & -21.7 & -0.1 & 1.0 & 35.3 & -0.4 & 0.9 & Fair \\
\hline $55,242.819$ & 0.654 & 12.1 & -0.3 & 1.0 & -9.9 & -0.4 & 0.9 & Fair \\
\hline $55,272.757$ & 0.877 & -16.7 & -0.1 & 1.0 & 29.8 & 0.7 & 0.9 & Fair \\
\hline $55,280.771$ & 0.937 & -24.2 & 0.1 & 1.0 & 39.8 & 0.5 & 0.9 & Fair \\
\hline $55,298.001$ & 0.066 & -17.0 & -0.3 & 1.0 & 29.7 & 0.5 & 0.9 & Fair \\
\hline $55,332.977$ & 0.326 & 18.7 & 0.1 & 1.0 & -17.3 & 0.4 & 0.9 & Fair \\
\hline $55,344.972$ & 0.416 & 21.3 & 0.2 & 1.0 & -20.9 & 0.1 & 0.9 & Fair \\
\hline $55,362.903$ & 0.549 & 18.7 & -0.1 & 1.0 & -17.5 & 0.5 & 0.9 & Fair \\
\hline $55,365.971$ & 0.572 & 17.4 & -0.4 & 0.9 & -16.7 & -0.1 & 0.6 & KPNO \\
\hline $55,375.901$ & 0.646 & 12.6 & -0.4 & 1.0 & -9.9 & 0.4 & 0.9 & Fair \\
\hline $55,464.869$ & 0.310 & 17.3 & -0.3 & 1.9 & -16.3 & 0.2 & 0.6 & KPNO \\
\hline $55,479.866$ & 0.421 & 21.3 & 0.2 & 1.0 & -20.9 & 0.2 & 0.9 & Fair \\
\hline $55,494.800$ & 0.533 & 19.3 & -0.2 & 1.0 & -18.9 & 0.0 & 0.9 & Fair \\
\hline $55,503.887$ & 0.601 & 16.1 & -0.1 & 1.0 & -14.2 & 0.4 & 0.9 & Fair \\
\hline
\end{tabular}


Table 4

(Continued)

\begin{tabular}{lcccccccc}
\hline \hline $\begin{array}{l}\text { Hel. Julian Date } \\
(\mathrm{HJD}-2,400,000)\end{array}$ & Phase & $\begin{array}{c}V_{1} \\
\left(\mathrm{~km} \mathrm{~s}^{-1}\right)\end{array}$ & $\begin{array}{c}(O-C)_{1} \\
\left(\mathrm{~km} \mathrm{~s}^{-1}\right)\end{array}$ & $\mathrm{Wt}_{1}$ & $\begin{array}{c}V_{2} \\
\left(\mathrm{~km} \mathrm{~s}^{-1}\right)\end{array}$ & $\begin{array}{c}(O-C)_{2} \\
\left(\mathrm{~km} \mathrm{~s}^{-1}\right)\end{array}$ & $\mathrm{Wt}_{2}$ & Source $^{\mathrm{a}}$ \\
\hline $55,514.937$ & 0.683 & 9.2 & -0.7 & 1.0 & -6.0 & 0.1 & 0.9 & Fair \\
$55,541.892$ & 0.884 & -17.8 & -0.2 & 1.0 & 30.4 & 0.0 & 0.9 & Fair \\
$55,549.862$ & 0.943 & -24.9 & -0.1 & 1.0 & 40.0 & 0.0 & 0.9 & Fair \\
$55,558.679$ & 0.009 & -24.9 & -0.2 & 1.0 & 40.1 & 0.2 & 0.9 & Fair \\
$55,575.829$ & 0.137 & -3.4 & -0.4 & 1.0 & 11.0 & 0.0 & 0.9 & Fair \\
$55,586.796$ & 0.219 & 9.5 & -0.1 & 1.0 & -5.2 & 0.5 & 0.9 & Fair \\
$55,591.827$ & 0.256 & 13.4 & -0.2 & 1.0 & -10.6 & 0.5 & 0.9 & Fair \\
$55,609.742$ & 0.390 & 20.9 & 0.2 & 1.0 & -20.6 & -0.1 & 0.9 & Fair \\
$55,622.732$ & 0.487 & 21.0 & 0.3 & 1.0 & -20.5 & 0.0 & 0.9 & Fair \\
\hline
\end{tabular}

Note. ${ }^{a}$ KPNO: Kitt Peak National Observatory; McD: McDonald Observatory; Fair: Fairborn Observatory.

Table 5

Orbital Elements and Related Parameters of 38 Cas

\begin{tabular}{lll}
\hline \hline Parameter & Wright \& Pugh (1954) & This Study \\
\hline$P$ (days) & 134.078 & $134.1301 \pm 0.0049$ \\
$T$ (HJD) & $2,429,000.433 \pm 0.266$ & $2,454,350.29 \pm 0.11$ \\
$e$ & $0.310 \pm 0.016$ & $0.2398 \pm 0.0014$ \\
$\omega_{1}(\mathrm{deg})$ & $188.2 \pm 2.1$ & $194.45 \pm 0.33$ \\
$K_{1}\left(\mathrm{~km} \mathrm{~s}^{-1}\right)$ & $19.92 \pm 0.3$ & $23.654 \pm 0.046$ \\
$K_{2}\left(\mathrm{~km} \mathrm{~s}^{-1}\right)$ & $\ldots$ & $31.440 \pm 0.052$ \\
$\gamma\left(\mathrm{km} \mathrm{s}^{-1}\right)$ & $1.10 \pm 0.3$ & $3.001 \pm 0.026$ \\
$m_{1} \sin ^{3} i\left(M_{\odot}\right)$ & $\ldots$ & $1.2163 \pm 0.0046$ \\
$m_{2} \sin ^{3} i\left(M_{\odot}\right)$ & $\ldots$ & $0.9150 \pm 0.0036$ \\
$a_{1} \sin i\left(10^{6} \mathrm{~km}\right)$ & 34.9 & $42.354 \pm 0.084$ \\
$a_{2} \sin i\left(10^{6} \mathrm{~km}\right)$ & $\ldots$ & $56.296 \pm 0.095$ \\
Standard error of a unit weight observation $\left(\mathrm{km} \mathrm{s}^{-1}\right)$ & $\ldots$ & 0.29 \\
\hline
\end{tabular}

0.4 , and 0.4 for the Fairborn, KPNO, and McDonald primary velocities, respectively. Velocities from the weaker secondary lines have weights of $0.2,0.1$, and 0.1 for the Fairborn, KPNO, and McDonald observations, respectively. The center-of-mass velocities of the four data sets agree to within $0.2 \mathrm{~km} \mathrm{~s}^{-1}$. A simultaneous solution of the appropriately weighted primary and secondary velocities produced a period of 42.3813 days.

In principle, the older literature velocities of the primary could improve the precision of the period and perhaps the other orbital elements. However, the vast majority of the measurements are from photographic plates that have significantly lower resolution than our observations. Even analyzing just the unblended primary velocities of Nadal et al. (1983), the resulting weight for those velocities is less than 0.01 , and so they do not significantly improve our solution. Therefore, we have chosen to list in Table 7 the double-line orbital solution determined with the new velocities from our three observatories. Figure 4 compares our primary and secondary velocities with the calculated velocity curves.

In addition to our elements, Table 5 lists those of Nadal et al. (1983). There is general agreement between the two solutions although the precision of our new orbital elements is improved by up to a factor of 40 . The two values of the systemic velocity differ by $1.2 \mathrm{~km} \mathrm{~s}^{-1}$, roughly a $2 \sigma$ result, so there is no strong evidence for a third body in the system. Of greater significance is the $11 \%$ decrease in the minimum mass of the primary, resulting mostly from a $3 \mathrm{~km} \mathrm{~s}^{-1}$ decrease in the semiamplitude of the secondary.

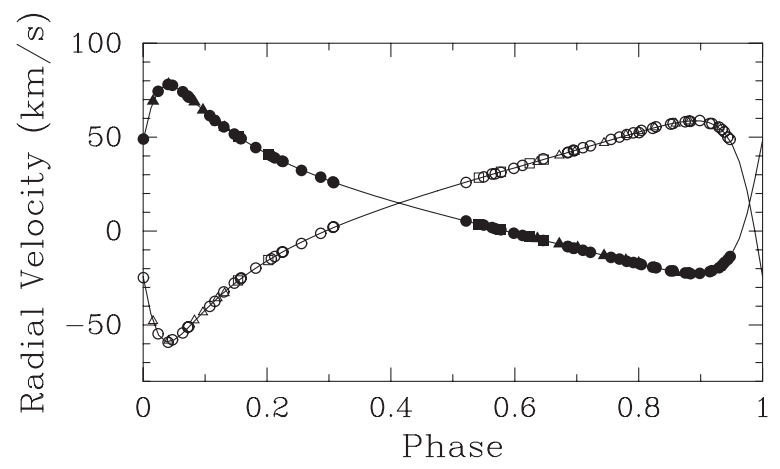

Figure 4. Radial velocities of HR 8467 compared with the computed velocity curves. Filled and open symbols represent the primary and secondary, respectively. Circles: Fairborn Observatory; triangles: KPNO; squares: McDonald Observatory. Zero phase is a time of periastron passage.

\section{SPECTRAL TYPES AND MAGNITUDE DIFFERENCE}

Strassmeier \& Fekel (1990) identified several luminositysensitive and temperature-sensitive line ratios in the 6430$6465 \AA$ A region. They employed those critical line ratios and the general appearance of the spectrum as spectral-type criteria. Unfortunately, for stars that are hotter than early-G spectral class, the line ratios in that wavelength region have little sensitivity to luminosity. However, the luminosity class may be determined by computing the absolute visual magnitude with the Hipparcos parallax and comparing that magnitude to evolutionary tracks or a table of canonical values for giants 
Table 6

Radial Velocities of HR 8467

\begin{tabular}{|c|c|c|c|c|c|c|c|c|}
\hline $\begin{array}{l}\text { Hel. Julian Date } \\
(\mathrm{HJD}-2,400,000)\end{array}$ & Phase & $\begin{array}{c}V_{1} \\
\left(\mathrm{~km} \mathrm{~s}^{-1}\right)\end{array}$ & $\begin{array}{l}(O-C)_{1} \\
\left(\mathrm{~km} \mathrm{~s}^{-1}\right)\end{array}$ & $\mathrm{Wt}_{1}$ & $\begin{array}{c}V_{2} \\
\left(\mathrm{~km} \mathrm{~s}^{-1}\right)\end{array}$ & $\begin{array}{c}(O-C)_{2} \\
\left(\mathrm{~km} \mathrm{~s}^{-1}\right)\end{array}$ & $\mathrm{Wt}_{2}$ & Source ${ }^{a}$ \\
\hline $52,965.659$ & 0.072 & 71.9 & 0.1 & 1.0 & -51.2 & 0.5 & 0.2 & Fair \\
\hline $53,170.900$ & 0.915 & -21.6 & 0.0 & 1.0 & 57.3 & -0.4 & 0.2 & Fair \\
\hline $53,171.901$ & 0.938 & -16.8 & 0.3 & 1.0 & 52.8 & 0.4 & 0.2 & Fair \\
\hline $53,283.756$ & 0.578 & 0.7 & 0.0 & 1.0 & 31.3 & -0.3 & 0.2 & Fair \\
\hline $53,288.766$ & 0.696 & -9.1 & -0.2 & 1.0 & 42.7 & -0.1 & 0.2 & Fair \\
\hline $53,296.726$ & 0.884 & -22.7 & -0.2 & 1.0 & 58.4 & -0.3 & 0.2 & Fair \\
\hline $53,302.680$ & 0.024 & 74.4 & -0.1 & 1.0 & -54.7 & 0.1 & 0.2 & Fair \\
\hline $53,303.665$ & 0.047 & 77.6 & 0.0 & 1.0 & -57.9 & 0.5 & 0.2 & Fair \\
\hline $53,310.668$ & 0.213 & 39.0 & -0.1 & 1.0 & -13.5 & -0.1 & 0.2 & Fair \\
\hline $53,314.644$ & 0.306 & 26.2 & 0.0 & 1.0 & 2.0 & 0.3 & 0.2 & Fair \\
\hline $53,330.696$ & 0.685 & -8.2 & -0.1 & 1.0 & 41.7 & -0.1 & 0.2 & Fair \\
\hline $53,341.666$ & 0.944 & -15.1 & 0.0 & 1.0 & 50.0 & 0.0 & 0.2 & Fair \\
\hline $53,353.612$ & 0.226 & 37.1 & 0.1 & 1.0 & -11.1 & -0.1 & 0.2 & Fair \\
\hline $53,538.929$ & 0.598 & -1.1 & -0.1 & 1.0 & 33.4 & -0.2 & 0.2 & Fair \\
\hline $53,552.969$ & 0.930 & -19.5 & -0.1 & 1.0 & 55.4 & 0.4 & 0.2 & Fair \\
\hline $53,566.780$ & 0.256 & 32.3 & -0.4 & 1.0 & -6.6 & -0.7 & 0.2 & Fair \\
\hline $53,603.847$ & 0.130 & 55.5 & -0.1 & 1.0 & -32.4 & 0.3 & 0.2 & Fair \\
\hline $53,627.771$ & 0.695 & -9.2 & -0.3 & 1.0 & 43.1 & 0.4 & 0.2 & Fair \\
\hline $53,634.765$ & 0.860 & -22.0 & -0.4 & 0.4 & 57.3 & -0.3 & 0.1 & KPNO \\
\hline $53,635.709$ & 0.882 & -22.8 & -0.3 & 0.4 & 58.5 & -0.1 & 0.1 & KPNO \\
\hline $53,637.753$ & 0.930 & -19.3 & 0.0 & 0.4 & 55.4 & 0.5 & 0.1 & KPNO \\
\hline $53,640.729$ & 0.000 & 49.0 & 0.0 & 1.0 & -24.8 & 0.2 & 0.2 & Fair \\
\hline $53,653.745$ & 0.308 & 25.8 & -0.2 & 1.0 & 2.3 & 0.4 & 0.2 & Fair \\
\hline $53,663.685$ & 0.542 & 3.6 & 0.0 & 0.4 & 28.5 & 0.4 & 0.1 & $\mathrm{McD}$ \\
\hline $53,666.660$ & 0.612 & -2.3 & -0.1 & 1.0 & 35.0 & 0.1 & 0.2 & Fair \\
\hline $53,679.629$ & 0.918 & -21.1 & 0.1 & 1.0 & 57.3 & 0.1 & 0.2 & Fair \\
\hline $53,689.586$ & 0.153 & 50.3 & 0.0 & 0.4 & -26.3 & 0.2 & 0.1 & $\mathrm{McD}$ \\
\hline $53,691.654$ & 0.202 & 40.9 & 0.0 & 0.4 & -15.4 & 0.1 & 0.1 & $\mathrm{McD}$ \\
\hline $53,692.591$ & 0.224 & 37.2 & -0.1 & 1.0 & -11.1 & 0.2 & 0.2 & Fair \\
\hline $53,728.595$ & 0.074 & 71.3 & 0.0 & 1.0 & -50.9 & 0.2 & 0.2 & Fair \\
\hline $53,875.923$ & 0.550 & 3.2 & 0.2 & 1.0 & 28.9 & 0.0 & 0.2 & Fair \\
\hline $53,888.875$ & 0.856 & -21.2 & 0.2 & 1.0 & 57.3 & -0.1 & 0.2 & Fair \\
\hline $53,928.939$ & 0.801 & -17.3 & 0.2 & 1.0 & 52.4 & -0.4 & 0.2 & Fair \\
\hline $54,006.737$ & 0.637 & -4.0 & 0.1 & 0.4 & 36.9 & -0.3 & 0.1 & KPNO \\
\hline $54,011.771$ & 0.755 & -14.0 & -0.2 & 1.0 & 48.8 & 0.3 & 0.2 & Fair \\
\hline $54,028.810$ & 0.157 & 49.3 & -0.1 & 1.0 & -25.4 & 0.1 & 0.2 & Fair \\
\hline $54,046.623$ & 0.578 & 0.7 & 0.0 & 0.4 & 31.6 & 0.0 & 0.1 & $\mathrm{McD}$ \\
\hline $54,048.590$ & 0.624 & -3.0 & 0.1 & 0.4 & 36.1 & 0.1 & 0.1 & $\mathrm{McD}$ \\
\hline $54,049.551$ & 0.647 & -5.0 & 0.0 & 0.4 & 38.1 & 0.0 & 0.1 & $\mathrm{McD}$ \\
\hline $54,054.723$ & 0.769 & -14.9 & 0.0 & 1.0 & 50.1 & 0.3 & 0.2 & Fair \\
\hline $54,076.674$ & 0.287 & 28.7 & 0.1 & 1.0 & -1.2 & -0.1 & 0.2 & Fair \\
\hline $54,267.952$ & 0.800 & -17.0 & 0.4 & 0.4 & 53.3 & 0.6 & 0.1 & KPNO \\
\hline $54,364.706$ & 0.083 & 68.8 & 0.2 & 0.4 & -47.5 & 0.4 & 0.1 & KPNO \\
\hline $54,366.764$ & 0.131 & 55.2 & -0.1 & 0.4 & -33.0 & -0.7 & 0.1 & KPNO \\
\hline $54,407.666$ & 0.097 & 64.5 & -0.1 & 0.4 & -43.2 & 0.0 & 0.1 & KPNO \\
\hline $54,408.663$ & 0.120 & 58.1 & 0.0 & 0.4 & -35.7 & 0.0 & 0.1 & KPNO \\
\hline $54,643.957$ & 0.672 & -6.8 & 0.2 & 0.4 & 40.3 & -0.2 & 0.1 & KPNO \\
\hline $54,729.967$ & 0.701 & -8.8 & 0.6 & 0.4 & 42.3 & -0.6 & 0.1 & KPNO \\
\hline $54,731.777$ & 0.744 & -13.0 & -0.1 & 0.4 & 47.0 & -0.4 & 0.1 & KPNO \\
\hline $55,320.953$ & 0.646 & -5.0 & -0.1 & 1.0 & 38.5 & 0.4 & 0.2 & Fair \\
\hline $55,330.908$ & 0.881 & -22.2 & 0.2 & 1.0 & 58.6 & 0.0 & 0.2 & Fair \\
\hline $55,340.870$ & 0.116 & 58.9 & -0.4 & 1.0 & -37.4 & -0.4 & 0.2 & Fair \\
\hline $55,359.827$ & 0.563 & 1.9 & 0.0 & 1.0 & 30.4 & 0.2 & 0.2 & Fair \\
\hline $55,368.988$ & 0.779 & -15.5 & 0.3 & 0.4 & 51.2 & 0.4 & 0.1 & KPNO \\
\hline $55,370.822$ & 0.823 & -19.2 & 0.0 & 1.0 & 54.7 & -0.1 & 0.2 & Fair \\
\hline $55,370.975$ & 0.826 & -19.5 & -0.1 & 0.4 & 55.3 & 0.2 & 0.1 & KPNO \\
\hline $55,382.899$ & 0.107 & 61.5 & 0.0 & 1.0 & -40.1 & -0.5 & 0.2 & Fair \\
\hline $55,411.885$ & 0.791 & -16.7 & 0.1 & 1.0 & 52.3 & 0.3 & 0.2 & Fair \\
\hline $55,442.813$ & 0.521 & 5.4 & 0.0 & 1.0 & 25.9 & -0.2 & 0.2 & Fair \\
\hline $55,444.813$ & 0.568 & 1.3 & -0.1 & 1.0 & 30.5 & -0.2 & 0.2 & Fair \\
\hline $55,450.813$ & 0.710 & -10.2 & -0.1 & 1.0 & 44.4 & 0.2 & 0.2 & Fair \\
\hline $55,453.813$ & 0.781 & -16.1 & -0.2 & 1.0 & 51.4 & 0.5 & 0.2 & Fair \\
\hline $55,454.813$ & 0.804 & -17.8 & 0.0 & 1.0 & 53.6 & 0.4 & 0.2 & Fair \\
\hline $55,455.813$ & 0.828 & -19.5 & 0.1 & 1.0 & 55.5 & 0.3 & 0.2 & Fair \\
\hline $55,456.813$ & 0.852 & -21.2 & -0.1 & 1.0 & 57.0 & -0.1 & 0.2 & Fair \\
\hline
\end{tabular}


Table 6

(Continued)

\begin{tabular}{lccccrrrl}
\hline \hline $\begin{array}{l}\text { Hel. Julian Date } \\
\text { HJD - 2,400,000) }\end{array}$ & Phase & $\begin{array}{c}V_{1} \\
\left(\mathrm{~km} \mathrm{~s}^{-1}\right)\end{array}$ & $\begin{array}{c}(O-C)_{1} \\
\left(\mathrm{~km} \mathrm{~s}^{-1}\right)\end{array}$ & $\mathrm{Wt}_{1}$ & $\begin{array}{c}V_{2} \\
\left(\mathrm{~km} \mathrm{~s}^{-1}\right)\end{array}$ & $\begin{array}{r}(O-C)_{2} \\
\left(\mathrm{~km} \mathrm{~s}^{-1}\right)\end{array}$ & $\mathrm{Wt}_{2}$ & Source $^{\mathrm{a}}$ \\
\hline $55,457.813$ & 0.875 & -22.3 & 0.0 & 1.0 & 58.2 & -0.2 & 0.2 & Fair \\
$55,458.813$ & 0.899 & -22.5 & 0.0 & 1.0 & 58.9 & 0.2 & 0.2 & Fair \\
$55,463.784$ & 0.016 & 69.2 & 0.3 & 0.4 & -47.8 & 0.4 & 0.1 & KPNO \\
$55,464.813$ & 0.040 & 78.1 & 0.0 & 1.0 & -59.2 & -0.2 & 0.2 & Fair \\
$55,464.843$ & 0.041 & 78.2 & 0.1 & 0.4 & -58.3 & 0.7 & 0.1 & KPNO \\
$55,465.812$ & 0.064 & 74.2 & 0.1 & 1.0 & -54.2 & 0.1 & 0.2 & Fair \\
$55,469.812$ & 0.158 & 49.3 & 0.1 & 1.0 & -24.9 & 0.3 & 0.2 & Fair \\
$55,470.812$ & 0.182 & 44.5 & 0.0 & 1.0 & -19.7 & 0.0 & 0.2 & Fair \\
$55,493.703$ & 0.722 & -11.3 & -0.2 & 1.0 & 45.4 & 0.1 & 0.2 & Fair \\
$55,502.725$ & 0.935 & -18.1 & 0.0 & 1.0 & 54.0 & 0.4 & 0.2 & Fair \\
$55,511.724$ & 0.147 & 51.7 & 0.1 & 1.0 & -27.7 & 0.3 & 0.2 & Fair \\
$55,534.565$ & 0.686 & -8.2 & 0.0 & 1.0 & 42.0 & 0.1 & 0.2 & Fair \\
$55,545.650$ & 0.948 & -13.5 & -0.1 & 1.0 & 48.8 & 0.7 & 0.2 & Fair \\
$55,556.635$ & 0.207 & 40.0 & -0.1 & 1.0 & -14.8 & -0.3 & 0.2 & Fair \\
\hline
\end{tabular}

Note. ${ }^{\text {a }}$ Fair: Fairborn Observatory; KPNO: Kitt Peak National Observatory: McD: McDonald Observatory.

Table 7

Orbital Elements and Related Parameters of HR 8467

\begin{tabular}{lll}
\hline \hline Parameter & Nadal et al. (1983) & \multicolumn{1}{c}{ This Study } \\
\hline$P($ days $)$ & 42.38113 & $42.38128 \pm 0.00011$ \\
$T(\mathrm{HJD})$ & $2,442,452.09 \pm 0.14$ & $2,454,276.4298 \pm 0.0036$ \\
$e$ & $0.616 \pm 0.011$ & $0.62344 \pm 0.00037$ \\
$\omega_{1}(\mathrm{deg})$ & $294.7 \pm 1.9$ & $294.161 \pm 0.054$ \\
$K_{1}\left(\mathrm{~km} \mathrm{~s}^{-1}\right)$ & $49.5 \pm 1.2$ & $50.331 \pm 0.031$ \\
$K_{2}\left(\mathrm{~km} \mathrm{~s}^{-1}\right)$ & $62.1 \pm 1.3$ & $58.897 \pm 0.059$ \\
$\gamma\left(\mathrm{km} \mathrm{s}^{-1}\right)$ & $13.7 \pm 0.5$ & $14.906 \pm 0.016$ \\
$m_{1} \sin ^{3} i\left(M_{\odot}\right)$ & 1.66 & $1.4782 \pm 0.0033$ \\
$m_{2} \sin ^{3} i\left(M_{\odot}\right)$ & 1.32 & $1.2633 \pm 0.0023$ \\
$a_{1} \sin i\left(10^{6} \mathrm{~km}\right)$ & 22.7 & $22.934 \pm 0.016$ \\
$a_{2} \sin i\left(10^{6} \mathrm{~km}\right)$ & 28.5 & $26.837 \pm 0.029$ \\
Standard error of a unit weight observation $\left(\mathrm{km} \mathrm{s}^{-1}\right)$ & $\ldots$ & 0.14
\end{tabular}

and dwarfs. Since such luminosity classes do not come from an examination of the spectra, we have identified them by the words dwarf, subgiant, etc., rather than by Roman numerals.

Spectra of our three binaries were compared with the spectra of a number of late-A, F, and G stars primarily from the lists of Abt \& Morrell (1995) and Fekel (1997). The reference star spectra were obtained at KPNO with the same telescope, spectrograph, and detector as our binary star spectra. To facilitate a comparison, various combinations of the reference-star spectra were rotationally broadened, shifted in radial velocity, appropriately weighted, and added together with a computer program developed by Huenemoerder \& Barden (1984) and Barden (1985) in an attempt to reproduce the binary spectra.

The resulting best spectrum addition model is used to determine the continuum intensity ratio of the binary components at $6430 \AA$, a wavelength that is about 0.6 of the way between the central wavelengths of the Johnson $V$ and $R$ bandpasses. If the two stars have very similar spectral types, this intensity ratio is also the luminosity ratio and, thus, can be converted directly into a magnitude difference. However, if the lines of the secondary are intrinsically stronger than those of the primary, as would be the case, for example, if the components were main-sequence stars of rather different spectral types, then the continuum intensity ratio results in a minimum magnitude difference.

\subsection{And}

Because 47 And is an Am star, our analysis of its spectrum is limited. Spectral classes for classical Am stars, determined from their hydrogen lines, range from A4 to F1 (Abt \& Morrell 1995). The spectra of these stars are peculiar because lines of some metallic elements such as iron and strontium are stronger than expected, compared to the hydrogen classification, while elements such as calcium and scandium are weaker (Abt \& Morrell 1995). There are no hydrogen lines in our limited $6430 \AA$ Avavelength region, and the iron and calcium abundance peculiarities vary from star to star, making it impossible to adequately characterize the combined spectrum of the binary components with our limited number of reference spectra.

Abt \& Morrell (1995) classified the combined spectrum of 47 And as an Am star with A1, F1, and F2 spectral classes for its $\mathrm{Ca} \mathrm{K}$, hydrogen, and metal lines, respectively. We have compared its red-wavelength spectrum with several reference star spectra and have the following comments.

Our spectra show that most lines of the two components of 47 And are nearly identical in strength and width. The Fe I lines of both components have the strength of an F2 star in accord with the classification of Abt \& Morrell (1995), while the Ca I lines in both components of 47 And are very weak, indicating a much earlier spectral class. Thus, we conclude that both components are Am stars. The evolutionary track comparison discussed in 
Section 7.1 indicates that both components are main-sequence stars.

From our various spectrum fits to the iron lines, we estimate a $V$ mag difference of $0.03 \pm 0.1$. This assumes that the surface abundances of the two stars are the same, which, because both components are Am stars, is not necessarily the case. However, the primary/secondary mass ratio of 47 And is 1.03, arguing for a near zero magnitude difference as well. In agreement with our result, Fletcher (1967) estimated a magnitude difference of $0.09 \pm 0.06$ for the components from iron lines in the blue region. Our result and that of Fletcher (1967) indicate that the slightly brighter star is the slightly more massive one. In the analysis that follows, we adopt a $V$ mag difference of 0.05 .

\subsection{Cas}

Cowley (1976), Cowley \& Bidelman (1979), and Abt (2009) are in complete agreement that the combined spectrum of $38 \mathrm{Cas}$ has a spectral type of F6 V. The reference stars HR 3262 [F6 V (Cowley 1976) and mean $[\mathrm{Fe} / \mathrm{H}]=-0.31$ (Taylor 2005)] and HD 121320 [G5 V (Gray et al. 2003) and $[\mathrm{Fe} / \mathrm{H}]=$ -0.31 (Gray et al. 2003)] produce a very good fit to the lines of the primary and secondary, respectively. Thus, we conclude that the components of 38 Cas are slightly metal poor relative to the Sun.

The continuum intensity ratio of the secondary/primary is 0.280 , which results in a continuum magnitude difference of $1.4 \pm 0.2$ at $6430 \AA$. The secondary has a later spectral class than the primary, making this result a minimum magnitude difference. We adopt a $V$ magnitude difference of 1.6, which is similar to that given by Gray (1992) in his table of canonical properties and closely reproduces the observed $B-V$ color of the combined system. As discussed in Section 7.2, visual magnitudes from the reanalyzed Hipparcos parallax (van Leeuwen 2007) indicate that both components are on the main sequence.

\section{3. $H R 8467$}

Harlan (1974) classified the system as F8 IV while Cowley (1976) called it F6 V. From an inspection of our spectra of HR 8467 we find that despite the significant line strength difference of the components, the two stars are very similar in temperature. The best fit to its spectrum is with HR 5323 [F6 IV (Cowley 1976), mean $[\mathrm{Fe} / \mathrm{H}]=0.09$ (Taylor 2005)] for both components. This fit produces lines that are slightly too strong, suggesting that the iron abundance is closer to the solar value, perhaps 0.05 .

The continuum intensity ratio of the secondary/primary is 0.408 , which results in a continuum magnitude difference of $0.97 \pm 0.1$ at $6430 \AA$. Given the identical spectral classes for the two stars, we adopt a $V$ magnitude difference of 1.0. From our discussion in Section 7.3, we classify the primary as a subgiant and the secondary as a dwarf.

\section{DETERMINATION OF ASTROMETRIC ORBIT AND RESULTS}

\subsection{And}

An examination of the Hipparcos astrometric data for 47 And shows no evidence of any astrometric displacement of its photocenter. This is not surprising given a mass ratio close to unity and the very small $V$ magnitude difference, 0.05 , of the components.

\subsection{Cas}

For 38 Cas the Hipparcos observations show an astrometric wobble with an amplitude that exceeds the typical observational precision. The Hipparcos team (Perryman et al. 1997) therefore fitted the data with an orbital model. In addition to the five basic astrometric parameters (position, parallax, and proper motion), three orbital elements were determined. The remaining four, namely the orbital period, a time of periastron passage, the argument of the periastron, and the eccentricity, were adopted from the spectroscopic orbit of Wright \& Pugh (1954). That initial astrometric orbital solution was included in Part $\mathrm{O}$ of the Hipparcos Double and Multiple Systems Annex (Perryman et al. 1997). Jancart et al. (2005) also computed an astrometric orbit with the Hipparcos data.

The new Hipparcos data processing carried out by van Leeuwen (2007) also used the 1954 orbit. Unfortunately, the revised data for 38 Cas are completely useless because of an undocumented problem in the computation of the observational weights, so despite the overall general quality of the new reduction, we have had to use the older analysis for this system.

Our greatly improved spectroscopic orbit makes it of interest to recompute the astrometric orbit. Here we impose our spectroscopic values for the elements $P, e, \omega$, and $T$ (Table 5) on the Hipparcos data to obtain a new astrometric solution. The adoption of the parameters from the new spectroscopic orbit has no significant effect on the parallax and proper motion of the system. Of particular interest is the orbital inclination, which remains very high and essentially unchanged at a value of 88.3 , although its uncertainty is reduced to 4.9 . In the new solution the position angle of the line of nodes, $\Omega$, is increased by 3.2 to $163.4 \pm 4.7$. The biggest change to the elements is in the value of $a_{0}$ (the semimajor axis of the photocentric orbit), which at $6.83 \pm 0.12$ mas is increased by $10 \%$ ( 5 revised $\sigma)$.

The new orbital solution leads to a magnitude difference close to 2.1 for the components, which is about 0.5 mag larger than that adopted from spectroscopy, as discussed in Section 5.2. Such an increased magnitude difference would primarily affect the magnitude of the secondary, making it significantly fainter.

\section{3. $H R 8467$}

As with 47 And, we have examined the Hipparcos data for HR 8467 and found no evidence of any astrometric displacement of its photocenter. Although the magnitude difference for the components of HR 8467 is much larger, about 1 mag, than that of $47 \mathrm{And}$, the predicted maximum angular nodal separation of the HR 8467 components (Section 10) is only 3 mas. This leads to a maximum absolute photocentric shift that is well below the Hipparcos precision.

\section{BASIC PROPERTIES}

\subsection{And}

To determine the basic properties of 47 And, we use the Stefan-Boltzmann law. We begin by adopting a $V$ mag of 5.60 and a $B-V$ color of 0.276 mag from the Hipparcos catalog (Perryman et al. 1997). With our magnitude difference of 0.05 , the individual $V$ magnitudes are 6.33 and 6.38 for the primary and secondary, respectively. The new Hipparcos parallax reduction by van Leeuwen (2007) produces a value of $15.66 \pm 0.30$ mas and corresponds to a distance of $63.9 \pm$ $1.2 \mathrm{pc}$. At such a distance we assume that the interstellar extinction is negligible. The resulting absolute magnitudes are 


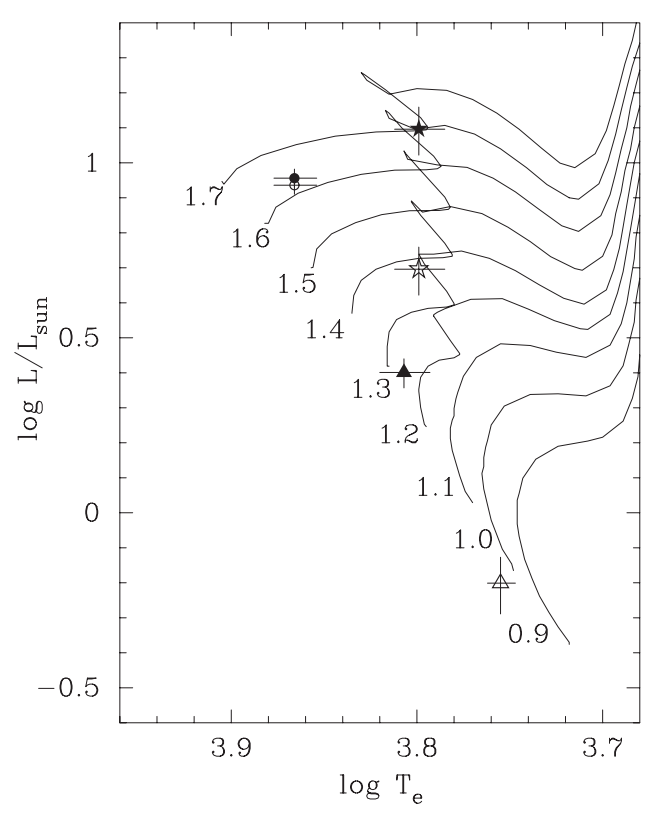

Figure 5. Positions of the components of 47 And (circles), 38 Cas (triangles), and HR 8467 (stars) are compared with the $0.9-1.7 M_{\odot}$ solar-abundance evolutionary tracks of Girardi et al. (2000) in a theoretical H-R diagram. The more massive component in each system corresponds to the filled symbol. Our estimated uncertainties are shown.

$M_{V}=2.30 \pm 0.06 \mathrm{mag}$ and $M_{V}=2.35 \pm 0.06 \mathrm{mag}$ for the primary and secondary, respectively. Given the similarity of the line strengths and masses, we adopt the mean $B-V$ color of 0.276 mag for both components. Then, from Table 3 of Flower (1996), we obtain the bolometric corrections and effective temperatures of the two components. The resulting luminosities of the primary and secondary are $L_{1}=9.0 \pm 0.5 L_{\odot}$ and $L_{2}=$ $8.6 \pm 0.5 L_{\odot}$, respectively, while the radii are $R_{1}=1.9 \pm$ $0.1 R_{\odot}$ and $R_{2}=1.8 \pm 0.1 R_{\odot}$, respectively. The small parallax uncertainty means that the luminosity uncertainties primarily result from the uncertainties associated with the individual magnitudes by way of the magnitude difference. The uncertainties of the radii are dominated by the effective temperature uncertainties, estimated to be $\pm 200 \mathrm{~K}$ for both components, which result from spectral type and temperature calibration uncertainties.

In Figure 5, we compare our estimated effective temperatures and luminosities of 47 And with the solar abundance evolutionary tracks of Girardi et al. (2000). Both stars are on the main sequence. Even if the abundance anomalies of the Am stars make them appear to be metal rich, as a result of the surface enhancement of iron, the components will still be on the main sequence. Relative to a solar composition, metal rich tracks are shifted to cooler temperatures and lower luminosities for the same masses. When compared to such tracks (Girardi et al. 2000), the stars are closer to the main sequence but of higher mass.

\subsection{Cas}

As we did for 47 And, we estimate the basic properties of 38 Cas by using the Stefan-Boltzmann law. We first adopt a $V$ mag of 5.82 and a $B-V$ color of 0.489 mag from the Hipparcos catalog (Perryman et al. 1997). If $\Delta V$ is $1.6 \mathrm{mag}$, then the magnitudes of the primary and secondary are 6.04 and $7.64 \mathrm{mag}$, respectively. The system parallax of $34.51 \pm 0.037$ mas (van Leeuwen 2007) results in a distance of $29.0 \pm 0.3 \mathrm{pc}$, and so we conclude that there is no reddening for this very nearby star. The components' absolute magnitudes are $M_{V}=3.7 \pm 0.1$ and $M_{V}=5.3 \pm 0.2$ for the primary and secondary, respectively. From Johnson (1966) we assume $B-V$ colors of 0.47 and 0.66 for the two components. Table 3 of Flower (1996) then provides the bolometric corrections and effective temperatures that we adopt for the stars. This produces luminosities of $L_{1}=$ $2.5 \pm 0.2 L_{\odot}$ and $L_{2}=0.6 \pm 0.1 L_{\odot}$ and radii of $R_{1}=$ $1.3 \pm 0.1 R_{\odot}$ and $R_{2}=0.82 \pm 0.08 R_{\odot}$ for the primary and secondary, respectively. The excellent precision of the parallax means that the uncertainties in the luminosities and radii are dominated by the magnitude difference uncertainties and the effective temperature uncertainties with the latter estimated as $\pm 200 \mathrm{~K}$ for the primary and $\pm 100 \mathrm{~K}$ for the secondary.

Our estimated temperatures and luminosities for the two stars are plotted in an H-R diagram and compared to the solarabundance evolutionary tracks of Girardi et al. (2000). The position of the two components in the H-R diagram indicates that both stars are dwarfs. This conclusion is not changed if, as we have found, 38 Cas is slightly metal poor relative to the Sun. Tracks for a more metal poor composition would be shifted to hotter temperatures and higher luminosities for the same masses. This would bring the computed masses of 1.22 and $0.92 M_{\odot}$ into better agreement with evolutionary track masses.

\section{3. $H R 8467$}

Once again, a determination of the basic properties of the components begins with the adoption of the $V$ magnitude and $B-V$ color of the HR 8467 system, which from the Hipparcos catalog (Perryman et al. 1997) are 6.39 and 0.498 , respectively. Using our $V$ magnitude difference of 1.0 , we compute individual apparent $V$ magnitudes of 6.75 and 7.75 . The new reduction of the Hipparcos parallax produces a value of $11.23 \pm 0.71$ mas (van Leeuwen 2007), which corresponds to a distance of $89.0 \pm 5.8 \mathrm{pc}$. At such a distance we assume that the system is not significantly reddened. We then combine the apparent magnitudes and the parallax to obtain absolute magnitudes $M_{V}=2.0 \pm 0.2$ and $M_{V}=3.0 \pm 0.2$ for the primary and secondary, respectively. We next adopt the same $B-V$ color of 0.498 for both stars, and use Table 3 of Flower (1996) to obtain the bolometric corrections and effective temperatures of the two components. The resulting luminosities of the primary and secondary are $L_{1}=12.5 \pm 2.0 L_{\odot}$ and $L_{2}=5.0 \pm 0.8 L_{\odot}$, respectively, while the radii are $R_{1}=3.0 \pm 0.3 R_{\odot}$ and $R_{2}=$ $1.9 \pm 0.2 R_{\odot}$, respectively. The uncertainties in the computed quantities are dominated by the parallax uncertainty plus, to a lesser extent, the effective temperature uncertainty, which is estimated to be $\pm 200 \mathrm{~K}$.

In Figure 5, from their positions in the H-R diagram compared to the solar-abundance evolutionary tracks of Girardi et al. (2000), the primary appears to be just beginning its evolution across the Hertzsprung gap or is perhaps at the very end of its main-sequence lifetime. The fainter secondary, on the other hand, is still on the main sequence.

\section{ECLIPSE SEARCHES}

Both 38 Cas and HR 8467 have large minimum masses, but their orbital periods of 134.130 and 42.3813 days, respectively, make it unlikely that eclipses occur. However, for 38 Cas the orbital inclination from our astrometric solution is very high, 88.3 , although that value has a relatively large uncertainty of 4.9. As luck would have it, both of these binaries were used 
as photometric comparison stars in a long-term program to search for luminosity cycles in solar-type stars. See Fekel et al. (2011) and references therein for a general discussion of the observations and their reduction.

Using eclipse ephemerides from our spectroscopic orbital elements, G. Henry (2011, private communication) examined 11 seasons of photometry of 38 Cas and 15 seasons of photometry of HR 8467. The long time baseline of over a decade for each star resulted in 893 observations of 38 Cas and 711 observations of HR 8467 that were well distributed in orbital phase. Despite the long orbital periods, the photometric observing cadence was such that several data points fell within the predicted time of eclipse that was closer to periastron for each of the two systems. Henry reports that the mean of those potential eclipse points was within $0.001 \mathrm{mag}$ of the mean for the rest of the data set. Thus, we find no evidence for eclipses in either system.

\section{CIRCULARIZATION AND SYNCHRONIZATION}

The two main theories of orbital circularization and rotational synchronization (e.g., Zahn 1977; Tassoul \& Tassoul 1992) disagree significantly on absolute timescales but do agree that synchronization should occur first. Matthews \& Mathieu (1992) examined 62 spectroscopic binaries with A-type primaries and periods less than 100 days. They concluded that all systems with orbital periods $\lesssim 3$ days have circular or nearly circular orbits. They also found that many binaries with periods in the range of 3-10 days have circular orbits. Surveying less massive stars, Duquennoy \& Mayor (1991) examined the multiplicity of solar-type stars in the solar neighborhood. They determined that systems with periods $\leqslant 10$ days had circular orbits but that longer period orbits are generally eccentric. With orbital periods ranging from 35 to 134 days, it is not surprising that the $47 \mathrm{And}$, 38 Cas, and HR 8467 systems have significantly eccentric orbits.

In an eccentric orbit, Hut (1981) has shown that the rotational angular velocity of a star will tend to synchronize with that of the orbital motion at periastron, a condition called pseudosynchronous rotation. We compute the pseudosynchronous rotation periods of our systems with Equation (42) of Hut (1981).

To help in assessing component pseudosynchronization in our three binaries, we have determined projected rotational velocities from our red-wavelength KPNO spectra with the procedure of Fekel (1997). For Am-type stars the measured line broadening was converted to a $v \sin i$ value. For stars with later spectral classes, macroturbulent broadening has been taken into account. Following Fekel (1997), for mid-F stars a value of $4 \mathrm{~km} \mathrm{~s}^{-1}$ was adopted, while for late-F and $\mathrm{G}$ stars a value of $3 \mathrm{~km} \mathrm{~s}^{-1}$ was used. To convert the $v \sin i$ values into equatorial rotational velocities, we assume, as is generally done, that the axes of the orbital and rotational planes are parallel, so the inclinations are equal.

\subsection{And}

To determine whether the components of 47 And are rotating pseudosynchronously, we first compute equatorial velocities from our projected rotational velocities. We then compare them with the predicted pseudosynchronous velocities.

For 47 And our $v \sin i$ values, averaged from five redwavelength spectra, are $15 \pm 2 \mathrm{~km} \mathrm{~s}^{-1}$ for both components. These identical values are at odds with the results of Abt \& Morrell (1995), who estimated very different values for the two components, $33 \mathrm{~km} \mathrm{~s}^{-1}$ for the primary and $<10 \mathrm{~km} \mathrm{~s}^{-1}$ for the secondary, from the measurement of the Mg II line at $4481 \AA$.
The minimum masses of the two components are small, both are much less than $1 M_{\odot}$. From our comparison with the solarabundance evolutionary tracks of Girardi et al. (2000) we adopt a mass of $1.65 M_{\odot}$ for the primary (Figure 5). Then that mass, combined with its $m \sin ^{3} i$ value (Table 3), results in an orbital inclination of $39^{\circ}$. If the rotational inclination is the same as that of the orbit, then the observed equatorial rotational velocities are $24 \mathrm{~km} \mathrm{~s}^{-1}$ for the primary and secondary. The pseudosynchronous rotation period of 47 And is 7.0 days. This value plus our computed radii from the Stefan-Boltzmann law produce pseudosynchronous rotational velocities of $13.4 \mathrm{~km} \mathrm{~s}^{-1}$ for the primary and secondary. Thus, the two components of 47 And are rotating about $80 \%$ faster than their pseudosynchronous values.

\subsection{Cas}

To determine if the components of 38 Cas are pseudosynchronously rotating, we initially compute their projected rotational velocities. From 13 spectra we determined $v \sin i$ values of $12.5 \pm 1$ and $5.5 \pm 2 \mathrm{~km} \mathrm{~s}^{-1}$ for the primary and secondary, respectively. From the astrometric solution the orbital inclination, and hence presumably the rotational inclination, is very close to $90^{\circ}$. Thus, we adopt the $v \sin i$ values as the equatorial rotational velocities. For 38 Cas we compute a pseudosynchronous rotation period of 99.3 days. The pseudosynchronous rotational velocities for the primary and secondary, computed with that period and our radii from the Stefan-Boltzmann law, are just 0.6 and $0.4 \mathrm{~km} \mathrm{~s}^{-1}$, respectively. With such a relatively large orbital period it is not surprising that the dwarf components are rotating much faster than pseudosynchronous.

\section{3. $H R 8467$}

Once again, to examine whether the rotational and orbital velocities of HR 8467 are linked pseudosynchronously, we first compute equatorial velocities from our measured projected rotational velocities. For HR 8467 our $v \sin i$ values, averaged from eight spectra, are $10.6 \pm 1$ and $9.5 \pm 2 \mathrm{~km} \mathrm{~s}^{-1}$, respectively. As shown in Figure 5, the position of the primary in the H-R diagram is somewhat evolved, and so we compare the position of the secondary with the evolutionary tracks, and adopt a mass of $1.38 M_{\odot}$. That value, combined with the $m \sin ^{3} i$ value of the secondary, results in an orbital inclination of $76^{\circ}$. If the axes of the rotational and orbital inclinations are parallel, then the observed equatorial rotational velocities are 10.9 and $9.8 \mathrm{~km} \mathrm{~s}^{-1}$.

The pseudosynchronous rotation period of HR 8467 is 9.4 days. This value plus our computed radii from the Stefan-Boltzmann law produce pseudosynchronous rotational velocities of 16.1 and $10.2 \mathrm{~km} \mathrm{~s}^{-1}$ for the primary and secondary, respectively. Thus, while the primary is rotating more slowly than its pseudosynchronous velocity, the secondary has achieved pseudosynchronous rotation.

\section{CONCLUSIONS}

We have determined improved orbital elements for three double-lined spectroscopic binaries, 47 And, 38 Cas, and HR 8467. With our detection of the secondary component of 38 Cas we have enhanced the utility of that system for the determination of its basic properties. A new astrometric solution of the Hipparcos astrometric data confirms that $38 \mathrm{Cas}$ is seen nearly edge on. Searches for eclipses in 38 Cas and HR 8467 have proved to be negative. Both components of 47 And are Am main-sequence stars, while our spectral types for 38 Cas are F6 dwarf and G5 dwarf for its primary and 
secondary, respectively. For HR 8467 we determined spectral types of F6 subgiant and F6 dwarf for the components. The primary is likely just beginning to traverse the Hertzsprung gap. The evolved primary of HR 8467 is rotating more slowly than its pseudosynchronous velocity, while its secondary is rotating pseudosynchronously, The components of the other two binaries are rotating significantly faster than their predicted pseudosynchronous velocities.

From the reanalyzed Hipparcos parallaxes (van Leeuwen 2007) and adopted masses of the three systems, we have estimated the maximum angular separations of the components. The orbits are eccentric, and so we determined maximum nodal separations (e.g., McAlister 1976; Halbwachs 1981), of 6, 28, and 3 mas, for 47 And, 38 Cas, and HR 8467, respectively. These separations are within the scope of modern optical interferometers. Thus, when our spectroscopic results are complemented with high-quality interferometric results, accurate three-dimensional orbits, masses, and distances for the systems will follow.

The referee's comments on our paper are appreciated and helped to make some of our explanations clearer. We thank Gregory Henry for examining his photometric data and communicating his results to us. The help of Daryl Willmarth in support of the KPNO coudé feed observations is appreciated. The research at Tennessee State University was supported in part by NASA, NSF, Tennessee State University, and the state of Tennessee through its Centers of Excellence program.

\section{REFERENCES}

Abt, H. A. 1961, ApJS, 6, 37

Abt, H. A. 1973, ApJS, 26, 472

Abt, H. A. 2009, ApJS, 180, 117

Abt, H. A., \& Morrell, N. I. 1995, ApJS, 99, 135

Abt, H. A., \& Moyd, K. I. 1973, ApJ, 182, 809

Adams, W. S., Joy, A. H., \& Sanford, R. F. 1924, PASP, 36, 137

Barden, S. C. 1985, ApJ, 295, 162

Barker, E. S., Evans, D. S., \& Laing, J. D. 1967, R. Obs. Bull., 130, 355

Boden, A. F., Torres, G., \& Latham, D. W. 2006, ApJ, 644, 1193

Cowley, A. P. 1976, PASP, 88, 95

Cowley, A. P., \& Bidelman, W. P. 1979, PASP, 91, 83

Cunha, M. S., et al. 2007, A\&AR, 14, 217

Duquennoy, A., \& Mayor, M. 1991, A\&A, 248, 485

Eaton, J. A., \& Williamson, M. H. 2004, Proc. SPIE, 5496, 710

Eaton, J. A., \& Williamson, M. H. 2007, PASP, 119, 886
Fekel, F. C. 1997, PASP, 109, 514

Fekel, F. C., Boden, A. F., Tomkin, J., \& Torres, G. 2009a, ApJ, 695, 1527

Fekel, F. C., Tomkin, J., \& Williamson, M. H. 2009b, AJ, 137, 3900

Fekel, F. C., Tomkin, J., \& Williamson, M. H. 2010, AJ, 139, 1579

Fekel, F. C., \& Williamson, M. H. 2010, AJ, 140, 1381

Fekel, F. C., Williamson, M. H., \& Henry, G. W. 2011, AJ, 141, 145

Fletcher, J. M. 1967, J. R. Astron. Soc. Canada, 61, 56

Flower, P. J. 1996, ApJ, 469, 355

Girardi, L., Bressan, A., Bertelli, G., \& Chiosi, C. 2000, A\&AS, 141, 371

Gray, D. F. 1992, The Observation and Analysis of Stellar Photospheres (Cambridge: Cambridge Univ. Press)

Gray, R. O., Corbally, C. J., Garrison, R. F., McFadden, M. T., \& Robinson, P. E. 2003, AJ, 126, 2048

Halbwachs, J. L. 1981, A\&AS, 44, 47

Harlan, E. A. 1974, AJ, 79, 682

Hartkopf, W. I., \& McAlister, H. A. 1984, PASP, 96, 105

Huenemoerder, D. P., \& Barden, S. C. 1984, BAAS, 16, 510

Hummel, C. A., et al. 2001, AJ, 121, 1623

Hut, P. 1981, A\&A, 99, 126

Jancart, S., Jorissen, A., Babusiaux, C., \& Pourbaix, D. 2005, A\&A, 442, 365

Jaschek-Corvalan, M., \& Jaschek, C. O. R. 1957, AJ, 62, 343

Johnson, H. L. 1966, ARA\&A, 4, 193

Jose, P. D. 1951, ApJ, 114, 370

Malaroda, S. 1975, AJ, 80, 637

Matthews, L. D., \& Mathieu, R. D. 1992, in ASP Conf. Ser. 32, IAU Colloq. 135, Complimentary Approaches to Double and Multiple Star Research, ed. H. A. McAlister \& W. I. Hartkopf (San Francisco, CA: ASP), 244

McAlister, H. A. 1976, PASP, 88, 317

McAlister, H. A. 1978, PASP, 90, 288

McCarthy, J. A., Sandiford, B. A., Boyd, D., \& Booth, J. 1993, PASP, 105, 881

Nadal, R., Carquillat, J. M., Pdeoussaut, A., \& Ginestet, N. 1983, A\&AS, 52, 293

Osawa, K. 1959, ApJ, 130, 159

Perryman, M. A. C., et al. 1997, A\&A, 323, L49

Plaskett, J. S., \& Young, R. K. 1919, J. R. Astron. Soc. Canada, 13, 59

Pourbaix, D., et al. 2004, A\&A, 424, 727

Quirrenbach, A. 2001, ARA\&A, 39, 353

Scarfe, C. D., Batten, A. H., \& Fletcher, J. M. 1990, Publ. Dom. Astrophys. Obs., 18,21

Strassmeier, K. G., \& Fekel, F. C. 1990, A\&A, 230, 389

Tassoul, J.-L., \& Tassoul, M. 1992, ApJ, 395, 259

Taylor, B. J. 2005, ApJS, 161, 444

Tomkin, J., \& Fekel, F. C. 2006, AJ, 131, 2652

Tomkin, J., \& Fekel, F. C. 2008, AJ, 135, 555

Torres, G., Andersen, J., \& Giménez, A. 2010, A\&AR, 18, 67

van Leeuwen, F. 2007, Hipparcos, The New Reduction of the Raw Data (Dordrecht: Springer)

Wilson, R. E., \& Joy, A. H. 1950, ApJ, 111, 221

Wolfe, R. H., Horak, H. G., \& Storer, N. W. 1967, in Modern Astrophysics, ed. M. Hack (New York: Gordon \& Breach), 251

Wright, K. O., \& Pugh, R. E. 1954, Publ. Dom. Astrophys. Obs., 9, 407

Zahn, J.-P. 1977, A\&A, 57, 383 